\title{
Quantum Monte Carlo and variational approaches to the Holstein model
}

\author{
Martin Hohenadler * Hans Gerd Evertz, and Wolfgang von der Linden \\ Institute for Theoretical Physics, Graz University of Technology, Petersgasse 16, A-8010 Graz, Austria
}

\begin{abstract}
Based on the canonical Lang-Firsov transformation of the Hamiltonian we develop a very efficient quantum Monte Carlo algorithm for the Holstein model with one electron. Separation of the fermionic degrees of freedom by a reweighting of the probability distribution leads to a dramatic reduction in computational effort. A principal component representation of the phonon degrees of freedom allows to sample completely uncorrelated phonon configurations. The combination of these elements enables us to perform efficient simulations for a wide range of temperature, phonon frequency and electron-phonon coupling on clusters large enough to avoid finite-size effects. The algorithm is tested in one dimension and the data are compared with exact-diagonalization results and with existing work. Moreover, the ideas presented here can also be applied to the many-electron case. In the one-electron case considered here, the physics of the Holstein model can be described by a simple variational approach.

PACS numbers: $63.20 . \mathrm{Kr}, 71.27 .+\mathrm{a}, 71.38 .-\mathrm{k}, 02.70 . \mathrm{Ss}$
\end{abstract}

\section{INTRODUCTION}

Quantum Monte Carlo (QMC) simulations for models with electron-phonon coupling are often limited in both system size and accessible parameter range by long autocorrelation times and large statistical errors. This makes it very difficult to study realistic models for, e.g., the high temperature superconductors or the manganites which exhibit colossal magnetoresistance. In both classes of materials electron-phonon interactions play an important role 1.2 Although classical treatments of phonons have been quite successful in certain situations $\stackrel{3.4}{=}$ e.g., at high-enough temperatures, quantum effects are expected to be relevant $\stackrel{2}{2}$ Consequently, it is highly desirable to develop a new, more efficient method to treat the phonon degrees of freedom quantum mechanically. The Holstein molecular-crystal model constitutes one of the simplest models for coupled electron-phonon systems, and therefore serves as an ideal testing ground for new approaches. Moreover, despite enormous theoretical efforts, even the physics of the Holstein model is still not completely understood.

The extensive use of QMC methods to study manybody problems is based on the fact that they can give quasiexact results (i.e., exact apart from statistical errors which can be made arbitrarily small, in principle). Over the years, several different QMC methods have been developed to study systems with electron-phonon coupling, such as the Holstein, $\frac{5}{\underline{5}}$ the Fröhlich $\underline{\underline{6}}$ or the SuSchrieffer-Heeger (SSH) model $\stackrel{7}{n}$ A very general QMC method for coupled fermion-boson models was developed

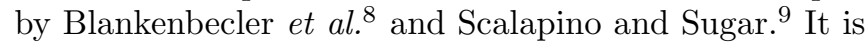
based on an analytic integration over the fermion degrees of freedom and a MC simulation of the resulting boson model. The simulation is performed using the grand-canonical ensemble and requires the evaluation of a fermion determinant involving a computation time which is proportional to the cube of the system size. Moreover, the method in its original form becomes unstable at low temperatures. While the simulations of Refs. 8 and 9 were restricted to one dimension, Levine and $\mathrm{Su}^{10,11}$ and, using a stabilized version of the same algorithm, Niyaz et al $\stackrel{12}{n}$ studied charge-density-wave formation and superconductivity in the two-dimensional Holstein model. A numerically faster method is the world-line algorithm developed by Hirsch et al ${ }^{13.14}$ based on a special breakup of the Hamiltonian and a fixed number of fermions. This results in configuration weights which are simple to evaluate allowing for much bigger system sizes. In the course of the simulation, both fermions and bosons are sampled simultaneously. The latter method has been successfully applied to the Holstein polaron problem and to the half-filled SSH and Holstein model 13.14.15.16.17 However the world-line algorithm is restricted to models in one spatial dimension or to the single-electron case in any dimension by the minus-sign problem. ${ }^{18}$ Scalettar et al $l^{19}$ applied a rather complicated so-called hybrid molecular dynamics algorithm to the two-dimensional Holstein model near half filling. This work was extended to the low-temperature regime by Noack et $a l^{20}$ Finally, Marsiglio ${ }^{21}$ developed a low-temperature QMC method to study the same model, also at half filling. De Raedt and Lagendijk ${ }^{22.23 .24}$ and Kornilovitch ${ }^{25}$ used an alternative approach based on Feynman's path-integral method, ${ }^{26}$ where the boson degrees of freedom are integrated out analytically and the resulting fermionic model is simulated using QMC. Although the method is limited to one electron or two electrons of opposite $\operatorname{spin}^{27}$ by the sign problem, it allows efficient simulations in one, two, and three dimensions even for small phonon frequencies near the adiabatic limit, and has also been used to investigate the Holstein model with dispersive phonons ${ }^{24}$ Also using Feynman's path integral, Kornilovitch and Pike ${ }^{28}$ developed a QMC method which exploits the conservation of the total quasimomentum of the system and allows the calculation of dynamical properties such as, e.g., the polaron band structure. Although the method is not restricted to a certain model or dimensionality of the system, it suffers from large statistical errors. Moreover, it is limited to the case of a single fermion at very low tem- 
perature, and exhibits a sign problem for nonzero total quasimomentum even in one dimension. Prokof'ev and Svistunov ${ }^{29}$ and Mischenko et al ${ }^{30}$ used QMC to directly sample the zero-temperature one-electron Green function of the Fröhlich polaron in imaginary time. The method allows calculations for an infinite system in three dimensions, but requires a convergent series for the electron propagator. While all but the last method mentioned so far make use of the Trotter-Suzuki approximation, 18 Kornilovitch ${ }^{31.32}$ developed a continuous-time algorithm that works in any dimension and allows calculations on infinite systems. It gives directly dynamical quantities such as the polaron spectrum and effective mass with very high accuracy. However, similar to previous work, ${ }^{28}$ it is restricted to the one-electron limit at very low temperatures. Moreover, calculations for small phonon frequencies and/or weak electron-phonon coupling are difficult and a sign problem appears for nonzero total quasimomentum. The projector QMC method ${ }^{18}$ in combination with a local updating of the phonon degrees of freedom has been used by Berger et al $l^{33}$ to investigate the Holstein-Hubbard model at various band fillings, and Green function QMC simulations for the halffilled Holstein model of spinless fermions have been performed by McKenzie et al ${ }^{34}$ Finally, the stochastic series expansion MC technique has been applied recently to an extended, one-dimensional Hubbard model with an electron-phonon interaction of the SSH type $\frac{35}{35}$ In contrast to other work, the phonons are treated in second quantization. Although the method allows simulations on large lattices in one dimension, it relies on an upper limit for the number of phonons at each site which makes it difficult to study the regime of small phonon frequencies and/or strong coupling.

In addition to the specific shortcomings of each method such as, e.g., the restriction to a single fermion, or to one spatial dimension, or to zero temperature, all previous simulations of the Holstein model were limited to some extent by autocorrelations. If the phonon degrees of freedom are not integrated out analytically, these correlations predominantly come from the free harmonicoscillator dynamics, especially in the adiabatic regime of small phonon frequency. This often leads to an enormous computational effort even for rather small systems and intermediate temperatures.

In this paper we first present a simple variational approach using a generalized form of the Lang-Firsov transformation which, in the one-electron case considered here, gives surprisingly good results and yields valuable insight into the mechanism of polaron formation. The full Hamiltonian resulting from the standard version of the canonical Lang-Firsov transformation is then used as the starting point for a QMC method which is free of any uncontrolled approximations. Due to the fact that the Lang-Firsov transformation contains the crucial electronic influences on the phonons, the Monte Carlo simulation for the phonon degrees of freedom can be based only on the purely phononic part of the trans- formed Hamiltonian. The electronic contributions can then be allowed for by reweighting of the probability distribution, corresponding to an exact treatment of the fermion degrees of freedom. This enables us to completely ignore the electronic weights in the updating process, and thereby dramatically reduce the computational effort. Finally, we introduce a principal component representation of the phonon coordinates, which allows exact sampling of the phonons and avoids all autocorrelations.

The paper is organized as follows. We briefly review the Holstein model in Sec. III In Sec. III we discuss the aforementioned transformations of the Hamiltonian. Section IV] is devoted to the variational polaron approach, while the QMC method for the Lang-Firsov transformed model is presented in Sec. $\nabla$ Section $\nabla]$ describes the reweighting method, and in Sec. VII the representation of the phonons in principal components is introduced. Results obtained with the given methods are presented in Secs. VIII and IX Finally, Sec. X contains our conclusions.

\section{THE HOLSTEIN MODEL}

The Holstein model has been introduced in the 1950's,,$\frac{5}{5}$ and describes a system of tight-binding conduction electrons coupled to a dispersionless phonon mode. If we express the phonon operators in terms of their natural units, the Hamiltonian takes the form

$$
\begin{aligned}
H & =K+P+I, \\
K & =-t \sum_{\langle i j\rangle \sigma} c_{i \sigma}^{\dagger} c_{j \sigma}, \\
P & =P_{\mathrm{p}}+P_{\mathrm{x}}=\frac{\omega}{2} \sum_{i}\left(\hat{p}_{i}^{2}+\hat{x}_{i}^{2}\right), \\
I & =-\alpha \sum_{i} \hat{n}_{i} \hat{x}_{i} .
\end{aligned}
$$

Here $c_{i \sigma}^{\dagger}\left(c_{i \sigma}\right)$ creates (annihilates) an electron of spin $\sigma$ at lattice site $i, \hat{x}_{i}$ and $\hat{p}_{i}$ denote the displacement and momentum of a harmonic oscillator at site $i$, and $\hat{n}_{i}=\sum_{\sigma} \hat{n}_{i \sigma}$ with $\hat{n}_{i \sigma}=c_{i \sigma}^{\dagger} c_{i \sigma}$. The last term $I$ describes the local coupling of the dispersionless Einstein phonons to the electron density $\hat{n}_{i}$. In the first term, the symbol $\langle i j\rangle$ denotes a summation over all nearest-neighbor hopping pairs $(i, j)$ and $(j, i)$. The parameters of the model are the hopping integral $t$, the phonon energy $\omega$, and the electron-phonon coupling constant $\alpha$. We introduce the commonly used dimensionless coupling constant $\lambda=\alpha^{2} /(\omega W)$, where $W=4 t d$ is the bare bandwidth in $d$ dimensions. We also define the dimensionless phonon frequency $\bar{\omega}=\omega / t$ and express all energies in units of $t$. Thus the model depends on two independent parameters, $\bar{\omega}$ and $\lambda$. Throughout this paper periodic boundary conditions in real space are assumed.

The Holstein model has been investigated intensively in the past, using a large variety of methods. Due 
to the large amount of literature available we restrict the discussion to the case of a single electron in an otherwise empty lattice with which this paper is concerned. The latter is generally known as the Holstein polaron problem and still constitutes a complicated manybody problem. Weak-coupling perturbation theory has been found to be accurate only for very small coupling strengths $\lambda$ when the phonon frequency is low $\underline{36}$ In the strong-coupling regime, an adiabatic small-polaron approximation ${ }^{5.37}$ has been found to work extremely well for small values of $\bar{\omega}$ (Ref. 38 ), while a perturbation theory based on the Lang-Firsov transformation ${ }^{39}$ gives accurate results for $\bar{\omega} \gg 1$ (Refs. 36 and 38 ). Discrepancies remain, however, in the regime of intermediate coupling and phonon frequency ${ }^{40}$ To bridge this gap, a lot of numerical work has been done using exact diagonalization (ED) methods, density-matrix renormalizationgroup (DMRG) studies, QMC methods and variational methods. ED is limited in the accessible parameter range, since it requires a truncation of the Hilbert space associated with the phonon degrees of freedom. With increasing electron-phonon coupling strength, for example, more and more phonon states have to be included to obtain converged results, ${ }^{36,38,41,42,43,44,45,46,47}$ which makes it difficult to study clusters of reasonable size in the strong or even intermediate coupling regime, especially for small phonon frequencies. At this point calculations based on DMRG set in, which use an optimized phonon basis to reduce the size of the Hilbert space ${ }^{48,49,50,51}$ Another possible approach are the so-called cluster methods which exploit exact information on small clusters to obtain approximate results for infinite systems Moreover, a number of variational methods have been developed which give very accurate results over a wide range of parameters 54.55 .56 .57 .58 .59 .60 .61 .62 As discussed in Sec. [ various QMC methods have been developed for the Holstein model. The polaron problem considered here has been investigated by Hirsch et al.,13.14 De Raedt and Lagendijk,$\stackrel{22,23.24}{\stackrel{ }{2} \text { Kornilovitch }, 25}$ Kornilovitch and Pike, ${ }^{28}$ Kornilovitch, ${ }^{31.32}$ and Mishchenko et al ${ }^{30}$ Finally, the Holstein polaron has also been studied in the infinite-dimensional limit using dynamical mean-field theory $\underline{\underline{63}}$

For the one-dimensional case, on which we will focus here, the general picture emerging from these investigations is as follows (see, e.g., Ref. 50). Starting from the noninteracting system $(\lambda=0)$ the electron is gradually dressed with a coherent multi-phonon cloud as the coupling increases. For $\lambda<1$ and $\lambda / \bar{\omega}<0.5$ the resulting quasiparticle remains in a Bloch-like state, with the phonon cloud giving rise to an increased effective mass. In the strong-coupling regime $(\lambda>1$ and $\lambda / \bar{\omega}>0.5)$ the electron becomes self-trapped by the induced lattice distortion and this object-trapped electron plus distortion - is usually called a small polaron. The transition from weak to strong coupling is continuous, $\stackrel{64}{=}$ and the term "large polaron" is often used to describe an electron dressed with a phonon cloud extending over more than one lattice site. The polaronic effects become more dominant as the phonon frequency approaches the adiabatic limit $\bar{\omega} \rightarrow 0$. In QMC simulations, small values of $\bar{\omega}$ introduce two very different time scales for the evolution of electrons and phonons, respectively. This gives rise to the problems mentioned above and, in fact, many QMC simulations have been done only for $\bar{\omega} \gtrsim 1$.

\section{EXTENDED LANG-FIRSOV TRANSFORMATION}

The canonical Lang-Firsov transformation ${ }^{39}$ has been used extensively to study the polaron problem. A wellknown approximation due to Holstein ${ }^{5}$ consists of replacing the transformed hopping term by its expectation value with respect to a zero-phonon state, thus neglecting phonon emission and absorption during the hopping process. This approximation, which we shall call the Holstein-Lang-Firsov (HLF) approximation, was found to give reliable results only in the strong-coupling and/or nonadiabatic limit $\lambda, \bar{\omega} \gg 1$ (Refs. 50, 43 and 44). More refined approaches based on strong-coupling perturbation theory provide an accurate description of the Holstein polaron over a large range of parameters $\frac{36.38}{}$ In the limit $\lambda=\infty$, the hopping term in Hamiltonian (11) can be neglected, and the Lang-Firsov transformation allows an exact solution of the resulting single-site problem. ${ }^{40}$ The transformation has also been used in combination with numerical methods $\frac{45,59.65}{}$ However we are not aware of any QMC simulation based on the transformed model.

We define the unitary operator

$$
U=e^{\nu}, \quad \nu=\mathrm{i} \sum_{i j} \gamma_{i j} \hat{n}_{i} \hat{p}_{j}
$$

where $i$ and $j$ run over lattice sites, and with real parameters $\gamma_{i j}$. $U$ as defined in Eq. (2) has the form of a translation operator. Given an electron at lattice site $i$, it corresponds to a displacement of the harmonic oscillators at all sites $j, j=1, \ldots, N$, by $\gamma_{i j}$. Hence the transformation describes a nonlocal phonon cloud surrounding an electron. This corresponds to the well-known concept of a large polaron, which extends over more than one lattice site. Using the transformation $\tilde{\hat{O}}=U \hat{O} U^{\dagger}$ we find for the transformed operators

$$
\begin{aligned}
& \tilde{\hat{x}}_{i}=\hat{x}_{i}+\sum_{j} \gamma_{i j} \hat{n}_{j} \quad, \quad \tilde{\hat{p}}_{i}=\hat{p}_{i} \\
& \tilde{c}_{i \sigma}^{\dagger}=c_{i \sigma}^{\dagger} e^{\mathrm{i} \sum_{j} \gamma_{i j} \hat{p}_{j}} \quad, \quad \tilde{c}_{i \sigma}=c_{i \sigma} e^{-\mathrm{i} \sum_{j} \gamma_{i j} \hat{p}_{j}} .
\end{aligned}
$$

Inserting these results into Eq. (11), the transformed 
Hamiltonian becomes

$$
\begin{aligned}
\tilde{H} & =\tilde{K}+P+\tilde{I}_{\mathrm{ep}}+\tilde{I}_{e e} \\
\tilde{K} & =-t \sum_{\langle i j\rangle \sigma} e^{\mathrm{i} \sum_{l}\left(\gamma_{i l}-\gamma_{j l}\right) \hat{p}_{l}} c_{i \sigma}^{\dagger} c_{j \sigma} \\
\tilde{I}_{\mathrm{ep}} & =\sum_{i j} \hat{n}_{j} \hat{x}_{i}\left(\omega \gamma_{i j}-\alpha \delta_{i j}\right) \\
\tilde{I}_{\mathrm{ee}} & =\sum_{i j} \hat{n}_{i} \hat{n}_{j}\left(\frac{\omega}{2} \sum_{l} \gamma_{l j} \gamma_{l i}-\alpha \gamma_{i j}\right) .
\end{aligned}
$$

Here the term $\tilde{I}_{\text {ep }}$ describes the coupling between electrons and phonons, while $\tilde{I}_{\mathrm{ee}}$ represents an effective electron-electron interaction. The Hamiltonian (4) will be the starting point for the variational polaron approach presented in the following section.

A more suitable approach for QMC simulations, however, is given by requiring that the electron-phonon terms cancel. Then $\gamma_{i j}=\gamma \delta_{i j}$ with $\gamma=\sqrt{\lambda W / \omega}$ and we obtain the standard Lang-Firsov transformation with the transformation operator

$$
U_{0}=e^{\nu_{0}} \quad, \quad \nu_{0}=\mathrm{i} \gamma \sum_{i} \hat{n}_{i} \hat{p}_{i}
$$

In contrast to the extended polaron cloud, defined by Eq. (2), now only the oscillator at the site of the electron is affected. The transformed Hamiltonian reads

$$
\begin{aligned}
\tilde{H} & =\tilde{K}+P+Q \\
\tilde{K} & =-t \sum_{\langle i j\rangle \sigma} c_{i \sigma}^{\dagger} c_{j \sigma} e^{\mathrm{i} \gamma\left(\hat{p}_{i}-\hat{p}_{j}\right)}, \\
Q & =-\frac{1}{2} \gamma^{2} \omega \sum_{i} \hat{n}_{i}^{2} .
\end{aligned}
$$

In the HLF or small-polaron approximation, the ground state of the transformed Hamiltonian is approximated by leaving all phonons in the ground state. It has been shown $^{50}$ that the small-polaron wave function becomes exact in the strong-coupling, nonadiabatic limit, and agrees qualitatively with the exact results also in the intermediate coupling regime. As discussed by Zhang et al., 50 the HLF approximation gives an overestimated shift of the equilibrium position of the oscillator in the presence of an electron, and does not reproduce the retardation effects when an electron hops onto a previously unoccupied site. Nevertheless, the local lattice distortion at the site of the electron contains the crucial impact of the electron on the lattice. Consequently, the transformed Hamiltonian (6) should be a good starting point to perform QMC simulations, which merely need to simulate small fluctuations around the zero-point motion. Indeed we will see in Sec. [X] that the expectation values of the phonon operators in the transformed Holstein model remain close to the results of the free-oscillator case over the whole range of the electron-phonon coupling. This makes sampling of the phonon degrees of freedom much more efficient. In principle, it would also be possible to develop a QMC algorithm starting with Hamiltonian (4), with the parameters $\gamma_{i j}$ being determined by the variational method discussed in the following section. However, we will see that the simple (local) Lang-Firsov transformation is already sufficient to obtain a very efficient $\mathrm{QMC}$ method.

From Eq. (6) it is obvious that the standard LangFirsov transformation on the one hand removes the electron-phonon coupling term, but on the other hand introduces complex valued hopping integrals which depend on the phonon momenta at the lattice sites involved in the hopping process. Moreover, for more than one electron in the system, the last term $Q$ introduces a Hubbard-like attractive interaction. In the case of the extended transformation, the electron-phonon interaction term cannot be eliminated entirely, the hopping term involves all phonon momenta $p_{i}$ as well as the parameters $\gamma_{i j}$, and the electron-electron interaction becomes long ranged. For these reasons it is expedient to base the QMC simulation on the local Lang-Firsov transformation (6).

For simplicity, we restrict ourselves in the present study to the case of a single electron so that $\hat{n}_{i} \hat{n}_{j}=\hat{n}_{i} \delta_{i j}$. The electron-electron interaction term in Hamiltonian (4) becomes

$$
\tilde{I}_{\mathrm{ee}}=\sum_{i} \hat{n}_{i}\left(\frac{\omega}{2} \sum_{l} \gamma_{l i}^{2}-\alpha \gamma_{i i}\right),
$$

while the corresponding term in the local Lang-Firsov transformation [last term of Hamiltonian (6)] reduces to

$$
Q \rightarrow-\lambda W / 2=-E_{\mathrm{P}} .
$$

Equations (77) and (8) both describe a shift in energy resulting from the original electron-electron interaction which is usually called the polaron binding energy $E_{\mathrm{P}}$.

\section{VARIATIONAL POLARON APPROACH}

Here we present a simple variational method which is based on the extended transformation discussed in the preceding section. Similar work along these lines using different transformations of the Hamiltonian as well as physically motivated wave functions can be found, for example, in Refs. 58, 62, and 60.61. As discussed above, the zero-phonon ansatz of the simple HLF approximation gives reliable results only in the limit of large $\bar{\omega}$ and $\lambda$. Whereas in HLF the parameter $\gamma$ of the Lang-Firsov transformation is chosen such that the electron-phonon coupling term $\tilde{I}_{\text {ep }}$ vanishes, in the variational polaron approach (VPA), we treat the $\gamma_{i j}$ as variational parameters which are determined by minimizing the ground-state energy in a zero-phonon basis. Like the HLF approximation, the VPA becomes exact in the weak-coupling limit $\lambda \rightarrow 0$ and in the nonadiabatic strong-coupling limit 
$\lambda, \bar{\omega} \rightarrow \infty$. We will see in Sec. VIII that the VPA also gives very accurate results for large phonon frequencies, $\bar{\omega} \gg 1$. This can easily be understood keeping in mind the discussion of the validity of the HLF approximation given in the preceding section. While the HLF ansatz overestimates the displacement of the local oscillator in the presence of an electron, the VPA determines this shift variationally. Moreover, the missing retardation effects in the response of the oscillator to an electron hopping onto the site become negligible for large phonon frequencies. Therefore, in addition to the cases stated above, the VPA also becomes exact in the nonadiabatic limit $\bar{\omega} \rightarrow \infty$. Although the limitations of the VPA in or near the adiabatic regime will clearly emerge when we discuss results in Sec.VIII it works surprisingly well if we keep in mind the simplicity of the method. Moreover, the reasons for the failure of the VPA in certain parameter regimes are physically clear and can easily be interpreted.

For translationally invariant systems the displacement fields satisfy the condition $\gamma_{i j}=\gamma_{|i-j|}$. Inserting this relation into Eq. (7), the expression inside the brackets becomes independent of the index $i$. For the single electron case with $\sum_{i} \hat{n}_{i}=1$ we have

$$
\tilde{I}_{\mathrm{ee}}=\frac{\omega}{2} \sum_{l} \gamma_{l}^{2}-\alpha \gamma_{0} .
$$

We solve the eigenvalue problem of the transformed Hamiltonian (4) in a zero-phonon basis for which we make the ansatz

$$
|l\rangle=c_{l \sigma}^{\dagger}|0\rangle \otimes \prod_{\nu}\left|\phi_{0}^{(\nu)}\right\rangle, \quad l=1, \ldots, N,
$$

where $\left|\phi_{0}^{(\nu)}\right\rangle$ denotes the ground state of the harmonic oscillator at site $\nu$. For simplicity, we restrict ourselves to one dimension, although the method can easily be extended to higher dimensions. The matrix elements of the hopping term in this basis are

$$
\begin{aligned}
\left\langle l|\tilde{K}| l^{\prime}\right\rangle & =-t_{l l^{\prime}} \prod_{\nu}\left\langle\phi_{0}^{(\nu)}\left|e^{\mathrm{i}\left(\gamma_{l \nu}-\gamma_{l^{\prime} \nu}\right) \hat{p}_{\nu}}\right| \phi_{0}^{(\nu)}\right\rangle \\
& =-t_{l l^{\prime}} \prod_{\nu} \int \mathrm{d} x \phi\left(x+\gamma_{l \nu}\right) \phi\left(x+\gamma_{l^{\prime} \nu}\right) \\
& =-t_{l l^{\prime}} e^{-\frac{1}{4} \sum_{\nu}\left(\gamma_{\nu}-\gamma_{\nu+l-l^{\prime}}\right)^{2}}
\end{aligned}
$$

where $t_{l l^{\prime}}=t \delta_{\left\langle l l^{\prime}\right\rangle}$ is nonzero for nearest-neighbor hopping pairs $l^{\prime}=l \pm 1$ and $\phi(x)$ is the harmonic-oscillator ground-state wave function in coordinate space. The matrix elements of the other terms of Hamiltonian (4) are easily evaluated yielding

$$
\begin{aligned}
\left\langle l|P| l^{\prime}\right\rangle & =\delta_{l l^{\prime}} \frac{\omega}{2}, \\
\left\langle l\left|\tilde{I}_{\mathrm{ep}}\right| l^{\prime}\right\rangle & =0, \\
\left\langle l\left|\tilde{I}_{\mathrm{ee}}\right| l^{\prime}\right\rangle & =\delta_{l l^{\prime}}\left(\frac{\omega}{2} \sum_{l} \gamma_{l}^{2}-\alpha \gamma_{0}\right) .
\end{aligned}
$$

The eigenstates of the transformed Hamiltonian (4) in the zero-phonon subspace, spanned by the basis states defined in Eq. (10), are

$$
\left|\psi_{k}\right\rangle=c_{k \sigma}^{\dagger}|0\rangle \otimes \prod_{\nu}\left|\phi_{0}^{(\nu)}\right\rangle
$$

with energy

$$
\begin{aligned}
E(k) & =E_{\mathrm{k}}+\frac{\omega}{2} N+\frac{\omega}{2} \sum_{l} \gamma_{l}^{2}-\alpha \gamma_{0} \\
E_{\mathrm{k}} & =-t \sum_{\delta= \pm 1} e^{\mathrm{i} k \delta} e^{-(1 / 4) \sum_{\nu}\left(\gamma_{\nu}-\gamma_{\nu+\delta}\right)^{2}}
\end{aligned}
$$

where $E_{\mathrm{k}}$ denotes the kinetic energy of the electron. Defining the Fourier-transformed parameters $\tilde{\gamma}_{q}$ as

$$
\tilde{\gamma}_{q}=\frac{1}{\sqrt{N}} \sum_{l} e^{\mathrm{i} q l} \gamma_{l}
$$

and using $\left(\gamma_{l} \in \mathbb{R}\right)$

$$
\sum_{\nu} \gamma_{\nu} \gamma_{\nu+\delta}=\sum_{q} \tilde{\gamma}_{q} \tilde{\gamma}_{-q} e^{\mathrm{i} q \delta}=\sum_{q} \tilde{\gamma}_{q}^{2} \cos q \delta,
$$

the kinetic energy can be written as

$$
\begin{aligned}
E_{\mathrm{k}} & =-t \sum_{\delta} e^{\mathrm{i} k \delta} e^{-(1 / 2) \sum_{q}(1-\cos q \delta) \tilde{\gamma}_{q}^{2}} \\
& =\tilde{\epsilon}_{0}(k) e^{-(1 / 2) \sum_{q}(1-\cos q) \tilde{\gamma}_{q}^{2}} \\
& =\tilde{\epsilon}_{\mathrm{eff}}(k)
\end{aligned}
$$

where $\tilde{\epsilon}_{0}(k)=-2 t \cos k$ is the tight-binding dispersion in one dimension. Using these results the ground-state energy finally becomes

$$
E(k)=\tilde{\epsilon}_{\mathrm{eff}}(k)+\frac{N \omega}{2}+\frac{\omega}{2} \sum_{q} \tilde{\gamma}_{q}^{2}-\frac{\alpha}{\sqrt{N}} \sum_{q} \tilde{\gamma}_{q} .
$$

The variational parameters $\tilde{\gamma}_{p}$ are determined by

$$
\frac{\partial E}{\partial \tilde{\gamma}_{p}}=-\tilde{\gamma}_{p} \tilde{\epsilon}_{\mathrm{eff}}(p)(1-\cos p)+\omega \tilde{\gamma}_{p}-\frac{\alpha}{\sqrt{N}} \stackrel{!}{=} 0 .
$$

The values for $\tilde{\gamma}_{p}$ which minimize the energy $E$ can then be obtained from

$$
\tilde{\gamma}_{p}=\frac{g}{\sqrt{N}} \frac{1}{\omega+\tilde{\epsilon}_{\mathrm{eff}}(p)(1-\cos p)} .
$$

As $\tilde{\epsilon}_{\text {eff }}$ depends on the set of parameters $\tilde{\gamma}_{p}$, this equation has to be solved self-consistently. Equation (20) has a typical random-phase approximation form, which is reasonable since a variational ansatz for the wave function of the untransformed Hamiltonian can be written as [see also Eq. (3)]

$$
U^{\dagger}\left|\psi_{k}\right\rangle=\frac{1}{\sqrt{N}} \sum_{j} e^{\mathrm{i} k j} c_{j \sigma}^{\dagger} e^{-\mathrm{i} \sum_{l} \gamma_{j l} \hat{p}_{l}}|0\rangle \otimes \prod_{\nu}\left|\phi_{0}^{(\nu)}\right\rangle .
$$


In addition to the total energy given by Eq. (18), we are also interested in the quasi-particle weight for momentum $k=0$, defined as

$$
\sqrt{z_{0}}=\left\langle 0\left|\tilde{c}_{k=0, \sigma}\right| \psi_{0}\right\rangle .
$$

Here $\left|\psi_{0}\right\rangle$ denotes the ground state with one electron of momentum $p=0$ and the oscillators in the ground state $\left|\phi_{0}\right\rangle$. Fourier transformation leads to

$$
\begin{aligned}
\sqrt{z_{0}} & =\frac{1}{N} \sum_{i j}\left\langle\phi_{0}\left|\left\langle 0\left|\tilde{c}_{i \sigma} c_{j \sigma}^{\dagger}\right| 0\right\rangle\right| \phi_{0}\right\rangle \\
& =\frac{1}{N} \sum_{i}\left\langle\phi_{0}\left|e^{-\mathrm{i} \sum_{k} \gamma_{i k} \hat{p}_{k}}\right| \phi_{0}\right\rangle \\
& =e^{-(1 / 4) \sum_{q} \tilde{\gamma}_{q}^{2}}
\end{aligned}
$$

where we have used the same steps as in Eq. (11).

Results obtained with the VPA will be presented in Sec. VIII

\section{MONTE CARLO FOR THE TRANSFORMED MODEL}

In contrast to the approximate variational approach presented in the preceding section, the QMC method discussed here is based on the exact Lang-Firsov transformation of the Holstein Hamiltonian. Therefore, the method is exact apart from statistical errors and Trotter discretization, as discussed in Sec. I1

\section{A. Partition function}

We begin with the evaluation of the partition function $\mathcal{Z}=\operatorname{Tr} e^{-\beta H}=\operatorname{Tr} e^{-\beta \tilde{H}}$, with $\tilde{H}$ given by Eq. (6). As indicated in the preceding section, for the case of a single electron, the last term in Hamiltonian (6) represents a constant energy shift. Moreover we can drop spin indices and are left with the Hamiltonian

$$
\tilde{H}=\tilde{K}+P-E_{\mathrm{P}} .
$$

The polaron binding energy given by Eq. (8) can be neglected during the QMC simulation, and needs only to be considered in calculating the total energy. For simplicity, we only consider the one-dimensional case here, although the generalization to higher dimensions is a simple matter. Using the Suzuki-Trotter decomposition we obtain ${ }^{18}$

$$
e^{-\beta \tilde{H}} \approx\left(e^{-\Delta \tau \tilde{K}} e^{-\Delta \tau P_{\mathrm{p}}} e^{-\Delta \tau P_{\mathrm{x}}}\right)^{L} \equiv \mathcal{U}^{L},
$$

where $\beta=\left(k_{\mathrm{B}} T\right)^{-1}$ and $\Delta \tau=\beta / L$. Splitting up the trace into a bosonic and a fermionic part and inserting $L$ complete sets of momentum eigenstates 18 we derive the approximation for the partition function

$$
\mathcal{Z}_{L}=\operatorname{Tr}_{\mathrm{f}} \int \mathrm{d} p_{1} \mathrm{~d} p_{2} \cdots \mathrm{d} p_{L}\left\langle p_{1}|\mathcal{U}| p_{2}\right\rangle \cdots\left\langle p_{L}|\mathcal{U}| p_{1}\right\rangle
$$

where $\mathrm{d} p_{\tau} \equiv \prod_{i} \mathrm{~d} p_{i, \tau}$. Each matrix element can be evaluated by inserting a complete set of phonon coordinate eigenstates $\int \mathrm{d} x_{\tau}\left|x_{\tau}\right\rangle\left\langle x_{\tau}\right|$. All $x_{\tau}$ integrals are of Gaussian form and can easily be carried out. The result is

$$
\begin{aligned}
\left\langle p_{\tau}\left|e^{-\Delta \tau P_{\mathrm{x}}}\right| p_{\tau+1}\right\rangle & =C^{N} e^{-\sum_{i}\left(p_{i, \tau}-p_{i, \tau+1}\right)^{2} /(2 \omega \Delta \tau)} \\
C & =\sqrt{\frac{2 \pi}{\omega \Delta \tau}}
\end{aligned}
$$

The normalization factor in front of the exponential has to be taken into account in the calculation of the total energy but cancels when we measure other observables. With the abbreviation $\mathcal{D} p=\mathrm{d} p_{1} \mathrm{~d} p_{2} \cdots \mathrm{d} p_{L}$ the partition function finally becomes

$$
\mathcal{Z}_{L}=C^{N L} \int \mathcal{D} p w_{\mathrm{b}} w_{\mathrm{f}}
$$

with the abbreviations

$$
w_{\mathrm{b}}=e^{-\Delta \tau S_{\mathrm{b}}}, \quad w_{\mathrm{f}}=\operatorname{Tr}_{\mathrm{f}} \Omega, \quad \Omega=\prod_{\tau} e^{-\Delta \tau \tilde{K}_{\tau}} .
$$

Here $\tilde{K}_{\tau}$ is $\tilde{K}$ with the phonon operators $\hat{p}_{i}$ replaced by the momentum $p_{i, \tau}$ on the $\tau$ th Trotter slice. The exponential of the hopping term may in the single-electron case be written as

$$
\begin{aligned}
e^{-\Delta \tau \tilde{K}_{\tau}} & =D_{\tau} \kappa D_{\tau}^{\dagger} \\
\kappa_{j j^{\prime}} & =\left(e^{\Delta \tau t h^{\mathrm{tb}}}\right)_{j j^{\prime}}, \quad\left(D_{\tau}\right)_{j j^{\prime}}=\delta_{j j^{\prime}} e^{\mathrm{i} \gamma p_{j, \tau}},
\end{aligned}
$$

where $h^{\text {tb }}$ is the $N \times N$ tight-binding hopping matrix. Thus we have the same matrix $\kappa$ for every time slice, which is transformed by the diagonal unitary matrices $D_{\tau}$. In our one-electron case, the fermionic weight $w_{\mathrm{f}}=\sum_{n}\langle n|\Omega| n\rangle$ is given by the sum over the diagonal elements of the matrix representation of $\Omega$ in the basis of one-electron states

$$
|n\rangle=c_{n}^{\dagger}|0\rangle \text {. }
$$

The bosonic action in Eq. (29) contains only classical variables and takes the form

$$
S_{\mathrm{b}}=\frac{\omega}{2} \sum_{i, \tau} p_{i, \tau}^{2}+\frac{1}{2 \omega(\Delta \tau)^{2}} \sum_{i, \tau}\left(p_{i, \tau}-p_{i, \tau+1}\right)^{2}
$$

where the indices $i=1, \ldots, N$ and $\tau=1, \ldots, L$ run over all lattice sites and Trotter times, respectively, with the periodic boundary conditions $p_{i, L+1}=p_{i, 1}$. It may also be written as

$$
S_{\mathrm{b}}=\sum_{i} \boldsymbol{p}_{i}^{\mathrm{T}} A \boldsymbol{p}_{i}
$$

with $\boldsymbol{p}_{i}=\left(p_{i, 1}, \ldots, p_{i, L}\right)$ and a "periodic" tridiagonal $L \times L$ matrix $A$ with nonzero elements

$$
A_{l, l}=\frac{\omega}{2}+\frac{1}{\omega \Delta \tau^{2}}, \quad A_{l, l \pm 1}=-\frac{1}{\omega \Delta \tau^{2}} .
$$


Since $\mathcal{Z}_{L}$ is a trace, it follows that $A_{1, L}=A_{L, 1}=$ $-1 /\left(\omega \Delta \tau^{2}\right)$.

At this stage, with the above result for the partition function, a QMC simulation of the transformed Holstein model would proceed as follows. In each MC step, a pair of indices $\left(i_{0}, \tau_{0}\right)$ on the $N \times L$ lattice of phonon momenta $p_{i, \tau}$ is chosen at random. At this site, a change $p_{i_{0}, \tau_{0}} \mapsto p_{i_{0}, \tau_{0}}+\Delta p$ of the phonon configuration is proposed. To decide upon the acceptance of the new configuration using the Metropolis algorithm, the corresponding weights $w_{\mathrm{b}} w_{\mathrm{f}}$ and $w_{\mathrm{b}}^{\prime} w_{\mathrm{f}}^{\prime}$ have to be calculated. Due to the local updating process, the change of the bosonic weight $\Delta w_{\mathrm{b}}=w_{\mathrm{b}}^{\prime}-w_{\mathrm{b}}$ can easily be obtained. In contrast, the fermionic weight requires the evaluation of the $L$ fold matrix product appearing in the definition of $\Omega$ in Eq. (29). The numerical effort for the calculation of $w_{\mathrm{f}}$ may be reduced by varying $\tau_{0}$ sequentially from 1 to $L$ instead of picking random values. In this case the calculation of the new fermionic weight, after the change of a single phonon momentum, can be reduced to only two matrix multiplications. Similar to other MC methods, a warm-up phase at the beginning of the simulation would be required for each set of parameters. An additional difficulty arises from the fact that, for the transformed model, the fermionic weight $w_{\mathrm{f}}$ is no longer strictly positive, even for the case of a single electron in one dimension. This is a consequence of the complex-valued hopping integrals, in contrast to simulations of, e.g., the Hubbard model, where a minus-sign problem occurs as a consequence of the Fermi statistics of the electrons 18 Here the average sign of $w_{\mathrm{f}}$ is smallest in the regime of small phonon frequency and low temperature. The sign problem is most pronounced for intermediate values of the electron-phonon coupling strength $\lambda$, where the cross over from a large to a small polaron occurs. However, in one dimension, it is not severe and reduces with increasing system size. For example, calculations in the most critical regime $\beta t=10, \bar{\omega}=0.1$, and $\lambda \approx 1$ have shown that $\langle\operatorname{sign}\rangle \equiv\left\langle w_{\mathrm{f}}\right\rangle /\left\langle\left|w_{\mathrm{f}}\right|\right\rangle$ increases from about 0.5 for $N=4$ to about 0.85 for $N=16$. Nevertheless, it remains to be seen to what extent the number of electrons and the dimensionality of the system affect the situation.

A related QMC approach to the original Holstein Hamiltonian (1) involves a very similar derivation ${ }^{8.9}$ to obtain the partition function, also in the one-electron limit. In fact the bosonic action $S_{\mathrm{b}}$ takes exactly the same form, with $p$ replaced by $x$. The main difference is the fermionic part of the partition function, contained in the matrix $\Omega$. While the Lang-Firsov transformation leads to a complicated hopping term, the standard approach for the untransformed model only includes the bare hopping operator given by Eq. (11). However, an interaction term $I$ [cf. Eq. (11)] appears, which contains the phonon coordinate $\hat{x}$. Hence the matrix $\Omega$ is replaced by

$$
\Omega^{\prime}=\prod_{\tau=1}^{L} \kappa V_{\tau}, \quad\left(V_{\tau}\right)_{j j^{\prime}}=\delta_{j j^{\prime}} e^{\Delta \tau \alpha x_{j, \tau}}
$$

and the path integral in the partition function [Eq. (28)] is over all coordinates $x$ instead of the momenta $p$. Apart from the fact that the coordinates $x$ are sampled instead of the phonon momenta, the QMC procedure for the untransformed model is identical to the simulation described above. We shall refer to this less sophisticated QMC method for the original Holstein Hamiltonian as the standard approach. For $\lambda=\alpha=0$, i.e., no electronphonon coupling, we have a set of $N$ independent harmonic oscillators, and both approaches are alike.

\section{B. Problems with the standard approach}

Let us briefly consider the noninteracting limit, in which the partition function can be written as $\mathcal{Z}_{L} \sim$ $\int \mathcal{D} p e^{-\Delta \tau S_{\mathrm{b}}}$. As discussed by Batrouni and Scalettar, 66 the difficulties encountered in QMC simulations, even for the simple case of a single $(N=1)$ harmonic oscillator, arise from the large condition number, i.e., the ratio of largest to smallest eigenvalue, of the bosonic action $S_{\mathrm{b}}$. For small values of $\Delta \tau$ this ratio is proportional to $(\omega \Delta \tau)^{-2}$ (Ref. 66), leading to autocorrelation times which grow quadratically with decreasing phonon frequency and the number $L$ of Trotter times. The physical reason for these correlations becomes obvious if we look at the bosonic action [Eq. (32)]. The latter can be thought of as being proportional to the energy of a given phonon configuration, $E=\Delta \tau S_{\mathrm{b}}$. While the first term corresponds to the kinetic energy of the oscillators, the second term describes a coupling in imaginary time, i.e., a pure quantum effect. As pointed out by Batrouni and Scalettar, 66 large changes of a single phonon degree of freedom, $p_{i, \tau}$ say, are very unlikely to be accepted due to the energy change proportional to $1 /(\omega \Delta \tau)$, which arises from the coupling to $p_{i, \tau \pm 1}$. However, a QMC simulation with only small local changes is extremely ineffective in sampling the relevant regions of phase space. Therefore, successive phonon configurations will be highly correlated. A possible solution might be the use of global updating schemes. Alternatively, the situation could be improved by transforming to the normal modes of the phonons, so that different step sizes can be used in updating each mode. We will see in Sec. VII that the principal component representation can indeed be used to completely eliminate these difficulties.

In addition to the above-mentioned autocorrelations, which are in fact independent of any electronic influences, standard simulations of the Holstein model become very difficult in the regime where polaron effects are large. This occurs at low temperatures, small phonon frequencies $\bar{\omega}<1$, and for intermediate or strong electronphonon coupling $\lambda \gtrsim 1$. Unfortunately, these are exactly the parameters of interest for simulations of real substances such as, e.g., the manganites. ${ }^{2}$ To illustrate the physical origin of these problems let us consider the case of a single electron in the Holstein model. As discussed in Sec. III in the polaronic regime, the electron 
drags with it a cloud of phonons which corresponds to a more or less localized lattice distortion. When the electron hops from site A (with a displaced oscillator corresponding to a small polaron), say, to a neighboring, previously unoccupied site B (with the oscillator in its undisplaced ground state) during a QMC simulation, the current phonon configuration is no longer energetically favorable. Clearly, the oscillator at site A has to return to its undisplaced ground state, while a corresponding phonon cloud has to be built up at site B. Such distortions of the lattice in the presence of an electron are large compared to the zero-point motion of the oscillator. On the other hand, only small changes of the current configuration will be accepted in the simulation. Consequently it takes an enormous number of single updates to obtain the new configuration in which the polaron has completely moved to site B. Obviously these polaron effects also give rise to strongly autocorrelated configurations, thereby dramatically increasing the numerical effort for the simulation. These problems due to polaron formation can be overcome by using the Lang-Firsov transformed model. The transformation separates the large displacements of the local oscillators, due to polaron effects, from the free-oscillator dynamics which correspond to vibrations around the shifted equilibrium positions. The quantities to be sampled, namely the phonon momenta $p$, only show a weak dependence on the electron-phonon coupling strength $\lambda$, in stark contrast to the coordinates $x$ in the original, untransformed model, whose expectation values grow linearly with $\lambda$ in the strong-coupling regime. In fact, the QMC results obtained for the transformed model (see also Sec. IX]) show that the statistical errors increase in the intermediate coupling regime $\lambda \approx 1$, but decrease again as we approach the strong-coupling limit. This is in perfect agreement with the fact that the the Lang-Firsov transformation diagonalizes the Hamiltonian (11) in the strong-coupling or atomic limit $\lambda \rightarrow \infty$ (see Sec. III), so that the QMC method based on the transformed model becomes more and more efficient as $\lambda$ increases.

\section{Observables}

Thermodynamic expectation values

$$
\langle O\rangle=\mathcal{Z}^{-1} \operatorname{Tr} \hat{O} e^{-\beta H}=\mathcal{Z}^{-1} \operatorname{Tr} \hat{\tilde{O}} e^{-\beta \tilde{H}}
$$

of observables $O$ are computed in the Lang-Firsov transformed representation via

$$
\langle O\rangle=\mathcal{Z}^{-1} \operatorname{Tr}_{\mathrm{f}} \int \mathrm{d} p\left\langle p\left|\hat{\tilde{O}} e^{-\beta \tilde{H}}\right| p\right\rangle .
$$

In this paper we are interested in the kinetic energy of the electron, the total energy, the mean square of the phonon momenta, and the momentum distribution $n(k) \equiv\left\langle\tilde{c}_{k}^{\dagger} \tilde{c}_{k}\right\rangle$ for various wave vectors $k$. We begin with the kinetic energy which is defined as

$$
E_{\mathrm{k}}=\langle K\rangle=-t \mathcal{Z}^{-1} \sum_{\langle i j\rangle} \operatorname{Tr}\left(c_{i}^{\dagger} c_{j} e^{\mathrm{i} \gamma\left(\hat{p}_{i}-\hat{p}_{j}\right)} e^{-\beta \tilde{H}}\right) .
$$

Using the same steps as in the derivation of the partition function (see Sec. $\nabla \mathrm{A}$ ), and absorbing the additional phase factor in a matrix $M=D_{1}^{\dagger} \Omega D_{1}$ [see Eq. [301] ], we find

$$
\begin{aligned}
E_{\mathrm{k}} & =-t \mathcal{Z}_{L}^{-1} \sum_{\langle i j\rangle} \int \mathcal{D} p w_{\mathrm{b}} \sum_{n}\left\langle n\left|M c_{i}^{\dagger} c_{j}\right| n\right\rangle \\
& =-t \mathcal{Z}_{L}^{-1} \sum_{\langle i j\rangle} \int \mathcal{D} p w_{\mathrm{b}}\langle j|M| i\rangle
\end{aligned}
$$

with one-electron states $|n\rangle$ as defined in Eq. (31). Using the matrix elements $M_{i j}=\langle i|M| j\rangle$ and the expectation values

$$
\langle O\rangle_{\mathrm{b}}=\frac{\int \mathcal{D} p w_{\mathrm{b}} O(p)}{\int \mathcal{D} p w_{\mathrm{b}}}
$$

with respect to the purely phononic weights $w_{\mathrm{b}}$ we obtain

$$
E_{\mathrm{k}}=-t \frac{\sum_{\langle i j\rangle}\left\langle M_{j i}\right\rangle_{\mathrm{b}}}{\sum_{i}\left\langle M_{i i}\right\rangle_{\mathrm{b}}} .
$$

Here we have already taken into account the reweighting method which will be discussed in detail in the following section. The total energy can be obtained from the thermodynamic relation $E=-\partial(\ln \mathcal{Z}) / \partial \beta$, with $\mathcal{Z}$ given by Eq. (28). The result is

$$
\begin{aligned}
E & =E_{\mathrm{k}}+\frac{\omega}{2} \sum_{i}\left\langle p_{i}^{2}\right\rangle+E_{\mathrm{ph}}^{\prime}-E_{\mathrm{P}} \\
E_{\mathrm{ph}}^{\prime} & =\frac{N}{2 \Delta \tau}-\frac{1}{2 \omega \Delta \tau^{2} L} \sum_{i, \tau}\left\langle\left(p_{i, \tau}-p_{i, \tau+1}\right)^{2}\right\rangle,
\end{aligned}
$$

where $E_{\mathrm{P}}$ is defined in Eq. (8) and the expectation values are calculated according to Eq. (43) given below. To compare with other work we subtract the ground-state energy of the phonons, $E_{0, \mathrm{ph}}=N \omega / 2$. Finally, $n(k)$ can be obtained using Fourier transformation. The result is

$$
n(k)=\frac{1}{N} \frac{\sum_{i j}\left\langle M_{i j}\right\rangle_{\mathrm{b}} e^{\mathrm{i} k(i-j)}}{\sum_{i}\left\langle M_{i i}\right\rangle_{\mathrm{b}}}
$$

with $k$ from the first Brillouin zone and the same matrix $M$ as in the case of the kinetic energy.

\section{REWEIGHTING}

In typical QMC simulations a large amount of the total computational effort goes into the calculation of the probability for the acceptance of a proposed change of 
the configuration. This probability is usually determined by the ratio of the weights of the new and the old configuration, as in the Metropolis algorithm used here. In the notation of Sec. $\mathbb{\nabla}$ this involves the calculation of $w_{\mathrm{b}}$ and $w_{\mathrm{f}}$ for the two configurations, $S$ and $S^{\prime}$ say, in every MC step. While the change in the bosonic weight, $w_{\mathrm{b}}\left(S^{\prime}\right) / w_{\mathrm{b}}(S)$, is easily calculated for the case of local updating, the fermionic weight given by Eq. (29) involves an $L$-fold matrix product of $N \times N$ matrices for each configuration. Although the numerical effort of the evaluation of such a matrix product can be reduced by scanning sequentially through the time slices (see Sec. $\nabla$ A) it still requires a lot of total computer time.

This can be avoided by reweighting of the probability distribution to be sampled. In the case considered here, this corresponds to taking into account only the change $w_{\mathrm{b}}\left(S^{\prime}\right) / w_{\mathrm{b}}(S)$ in the bosonic weight, and compensating for this by dividing the resulting expectation value by the expectation value of the fermionic weight $w_{\mathrm{f}}$, as has been used already in Eq. 40 leading generally to ratios of the form

$$
\langle O\rangle=\frac{\left\langle O w_{\mathrm{f}}\right\rangle_{\mathrm{b}}}{\left\langle w_{\mathrm{f}}\right\rangle_{\mathrm{b}}}
$$

where the subscript "b," defined in Eq. (39), indicates that the average is computed based on $w_{\mathrm{b}}$ only. Following this procedure, the fermionic weight is treated as part of the observables. The splitting into weight $w_{\mathrm{b}}$ and observable $O w_{\mathrm{f}}$ is sensible as long as the variance of $w_{\mathrm{f}}$ and $O w_{\mathrm{f}}$ is small, which is the case after the Lang-Firsov transformation. This approach has several additional advantages. With the reweighting method, the updating of the system does no longer require the calculation of $w_{\mathrm{f}}$ in every step, but only when measurements are performed. Compared to the usual QMC procedure described in Sec. $\nabla A$ this can save an enormous amount of computer time, allowing such simulations to be run on a standard personal computer instead of a high-performance supercomputer. Additionally, since the updating does no longer involve any electronic contributions, it becomes independent of the electron-phonon coupling strength $\lambda$. This allows the simultaneous measurement of observables for a whole set of values of $\lambda$ in a single MC run. For a given phonon configuration, the fermionic weight and the observables are measured and stored for each value of the coupling. This procedure is repeated until the required number of measurements has been made. At the end of the simulation an appropriate analysis of the measured values is made independently for each $\lambda$. In contrast, the QMC procedure without reweighting (see Sec. $\mathrm{VA}$ ) would require a separate run for each value of $\lambda$, including a warm-up phase to equilibrate the system for the current set of parameters. We will see in Sec. VII that in combination with the principal component representation, the phonon momenta $p$ can be sampled exactly, removing all autocorrelations. This avoids a warm-up phase, and measurements can be made after every Monte Carlo step. In this final, very efficient procedure, the calculation of

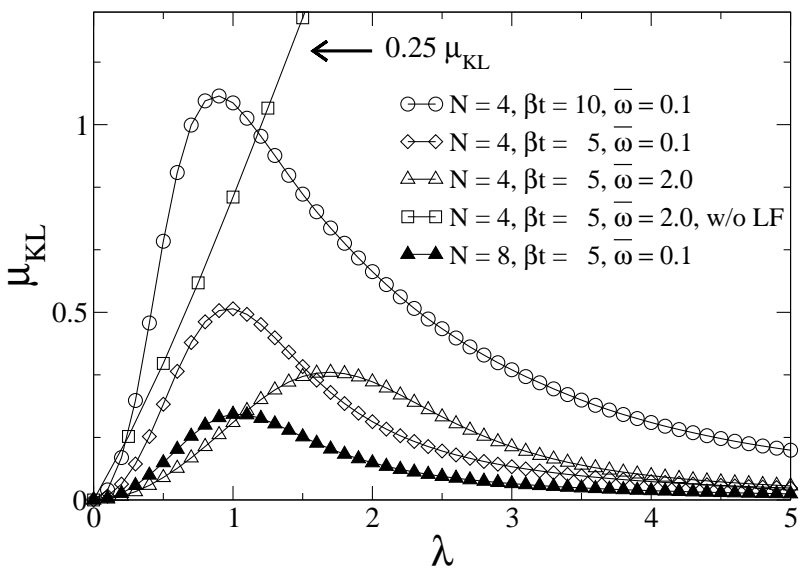

FIG. 1: Kullback-Leibler number $\mu_{\mathrm{KL}}$ as a function of electron-phonon coupling $\lambda$ for various sets of the parameters $N$ (number of sites), $\beta$ (inverse temperature) and $\bar{\omega}$. As indicated, the results for the untransformed model, denoted in the legend as "w/o LF," have been scaled by a factor 0.25 (see text). Error bars are smaller than the symbols shown, and lines are guides to the eye only.

$w_{\mathrm{f}}$ for measurements remains, and is then the most timeconsuming part of the calculation. Finally, we want to point out that, with the use of the reweighting method, the electronic degrees of freedom are treated exactly, i.e., they are not sampled in the course of the simulation.

As mentioned in Sec. $\mathrm{VA}$ the weight $w_{\mathrm{f}}$ for the transformed model is no longer strictly positive, so that it cannot be interpreted as a probability. The usual way to deal with such a sign problem is to split the weight into $w_{\mathrm{f}} \equiv\left|w_{\mathrm{f}}\right| \operatorname{sgn} w_{\mathrm{f}}$. Then $\left|w_{\mathrm{f}}\right|$ can be used as the weight of a given configuration in the updating process, while the sign is absorbed in the observables. The difference to the reweighting method presented here is that instead of the sign of $w_{\mathrm{f}}$, we treat the whole weight $w_{\mathrm{f}}$ as part of the observables.

Despite the obvious advantages of this approach, it is necessary to scrutinize whether reweighting does not lead to prohibitive statistical noise. If, for example, there was too small an overlap of the actual probability distribution with the one we are sampling with, the method would fail. In fact, our calculations have shown that for the untransformed model the reweighting method would fail at low temperatures and for critical values of the parameters $\bar{\omega}$ and $\lambda$.

The distance between two arbitrary probability distributions $\phi_{1}(x)$ and $\phi_{2}(x)$, each depending on a set of variables $x$, can be measured by the so-called KullbackLeibler number $\mu_{\mathrm{KL}}$ which is defined as 67

$$
\mu_{\mathrm{KL}}\left(\phi_{1}, \phi_{2}\right)=\int \mathrm{d} x \phi_{1}(x) \ln \frac{\phi_{1}(x)}{\phi_{2}(x)} .
$$

For $\phi_{1} \equiv \phi_{2}$ we have $\mu_{\mathrm{KL}}=0$, while for $\phi_{1} \neq \phi_{2}$ $\mu_{\mathrm{KL}}>0$. The fact that $\mu_{\mathrm{KL}}$ is a reasonable measure for the distance of two distributions is best illustrated 
by considering two Gaussian deviates $\phi_{1}, \phi_{2}$ with variance $\sigma^{2}$, centered at $x_{1}$ and $x_{2}$, respectively. In this case $\mu_{\mathrm{KL}}=\left(x_{1}-x_{2}\right)^{2} /\left(2 \sigma^{2}\right)$. For $\left|x_{1}-x_{2}\right|=\sqrt{2} \sigma$, where the two peaks begin to be distinguishable, we have $\mu_{\mathrm{KL}}=1$, while a large value of $\mu_{\mathrm{KL}} \simeq 10$, for example, corresponds to well-separated Gaussian distributions. Here we use the Kullback-Leibler number to investigate the applicability of the reweighting method. As long as the KullbackLeibler number is less than or comparable to 1, reweighting works well, while a Kullback-Leibler number strongly exceeding unity indicates severe problems. Two relevant distributions in our case are given by $\phi_{1}(\boldsymbol{p})=w_{\mathrm{b}}(\boldsymbol{p}) / \mathcal{Z}_{\mathrm{b}}$ and $\phi_{2}(\boldsymbol{p})=w_{\mathrm{b}}(\boldsymbol{p})\left|w_{\mathrm{f}}(\boldsymbol{p})\right| / \mathcal{Z}_{\mathrm{bf}}$, depending on the phonon configuration $\boldsymbol{p}$ (or $\boldsymbol{x}$ in the case of the untransformed Holstein model). $\mathcal{Z}_{\mathrm{b}}$ and $\mathcal{Z}_{\mathrm{bf}}$ are the normalization factors of the probability densities $\phi_{1}(\boldsymbol{p})$ and $\phi_{2}(\boldsymbol{p})$, and $w_{\mathrm{f}}$ has been replaced by its absolute value due to the aforementioned sign problem. Inserting these definitions into Eq. (44) we find $\mu_{\mathrm{KL}}=\ln \left\langle\left|w_{\mathrm{f}}\right|\right\rangle_{\mathrm{b}}-\left\langle\ln \left|w_{\mathrm{f}}\right|\right\rangle_{\mathrm{b}}$. Figure 1 shows results for $\mu_{\mathrm{KL}}$ for different parameters $\beta$, $\bar{\omega}$, and $N$. For $\lambda=0, w_{\mathrm{f}}$ is independent of the phonon configuration so that $\mu_{\mathrm{KL}}=0$. With increasing electronphonon coupling, the difference between the two distributions becomes larger. For an intermediate value of the electron-phonon coupling strength, $\mu_{\mathrm{KT}}$ takes on a maximum and approaches zero again in the strong-coupling limit $\lambda \rightarrow \infty$. This is exactly the behavior we would expect for the Lang-Firsov transformed model. For $\lambda=0$ the transformation has no effect and $w_{\mathrm{f}}$ is a constant, just as in the case of the untransformed model. In the intermediate coupling regime, the small-polaron picture mediated by the transformation is not correct as we have an extended (large) polaron in this region. However, as the coupling increases further, the polaron becomes smaller and for $\lambda=\infty$ it is known that the Lang-Firsov transformation diagonalizes the Holstein Hamiltonian (1). The dependence of $\mu_{\mathrm{KL}}$ on the temperature and the phonon frequency is also in perfect agreement with the physical picture of the Holstein polaron. As $\beta t$ increases, polaron effects become more prominent. The same effect occurs if we decrease $\bar{\omega}$, and in both cases the maximum of $\mu_{\mathrm{KL}}$ increases. In Fig. 11 the result for a system of eight lattice sites is also shown. The maximum in $\mu_{\mathrm{KL}}$ is clearly smaller than for the four-site cluster. Calculations for even larger clusters (not shown) reveal that the maximum in $\mu_{\mathrm{KL}}$ decreases further indicating that the overlap between $\phi_{1}$ and $\phi_{2}$ increases as $N \rightarrow \infty$. This behavior agrees well with the influence of finitesize effects in the transition region as pointed out before by Marsiglio ${ }^{36}$ As the system size increases, the cross over becomes smoother in agreement with the fact that the ground state of the Holstein polaron is an analytic function of the coupling $\lambda$ (Ref. 64). This point will be further illustrated in Sec. IX] To summarize, for all parameters shown in Fig. 11 the maximum of $\mu_{\mathrm{KL}}$ lies below $\mu_{\mathrm{KL}} \approx 1$, so that we can conclude that the two distributions are indeed very close and the reweighting method can be successfully applied.
We have also calculated the Kullback-Leibler number for the case of the untransformed model, denoted in Fig. 1 as "w/o LF," for which $\left|w_{\mathrm{f}}\right| \equiv w_{\mathrm{f}}$. The result has been divided by a factor 4 to allow a better representation in Fig. 11 The difference between $\phi_{1}$ and $\phi_{2}$ increases strongly with $\lambda$ and reaches large values of $\mu_{\mathrm{KT}}>10$ already in the intermediate coupling regime $1<\lambda<$ 2. Hence we cannot expect the reweighting method to work in this case. Finally we want to point out that the distance between $\phi_{1}$ and $\phi_{2}$ may not affect all observables in the same way. A detailed analysis for each observable $O$ would be based on the Kullback-Leibler distance of the marginal probability densities

$$
p_{\alpha}(o)=\int \mathrm{d} x p(o \mid x) p_{\alpha}(x)=\int \mathrm{d} x \delta(o-O(x)) p_{\alpha}(x)
$$

where $O(x)$ is the value of the observable for a given configuration $x$ and $\alpha=1,2$ for the two distributions under consideration.

In summary, the reweighting method, together with the Lang-Firsov transformation, allows us to sample a system of independent oscillators, while all the influence of the electrons is transferred to the observable, thereby strongly reducing the numerical effort. In order to obtain a reliable error analysis for observables calculated according to Eq. (43), the jackknife procedure ${ }^{68}$ has been applied.

\section{PRINCIPAL COMPONENT REPRESENTATION}

Although the reweighting method allows us, in principle, to skip enough sweeps between measurements to reduce autocorrelations to a minimum, the computational effort for these Monte Carlo updates can become the most time-consuming part of the simulation. Even though a single phonon update requires negligible computer time compared to the evaluation of the fermionic weight, in the critical parameter regime, an enormous number of such steps will be necessary between successive measurements. Moreover, reliable results can only be obtained when long enough Monte Carlo runs have been performed to see even the longest autocorrelation times. In this section, we present a principal component representation for the phonon degrees of freedom, which enables us to create completely uncorrelated samples of phonon configurations.

In order to illustrate the severe problem of autocorrelations with standard updates of the phonons, we have calculated the integrated autocorrelation time $\tau_{\text {int }}^{p}$ for the phonon momenta $p$. $\tau_{\text {int }}$ is a direct measure for the number of MC steps which have to be skipped between measurements in order to obtain uncorrelated data, and is usually given in units of sweeps. We define a sweep as $N$ times $L$ proposed local changes of the phonon configuration. For a four-site system, for example, with $\beta t=5$, $\lambda=2, \bar{\omega}=2$, and $\Delta \tau=0.05$ we find $\tau_{\text {int }}^{p} \approx 500$. This 
corresponds to an autocorrelation time of about $2 \times 10^{5}$ single MC steps. For smaller phonon frequencies, $\tau_{\text {int }}$ increases strongly. For $\bar{\omega}=1$ and the same $\Delta \tau$, the autocorrelation time is already $\approx 1700$ sweeps, which agrees quite well with the $(\omega \Delta \tau)^{-2}$ dependence of the correlations for $\lambda=0$ given in Sec. $\mathrm{VB}$. The dependence of $\tau_{\text {int }}^{p}$ on the coupling strength $\lambda$ is relatively weak, and we have found no systematic behavior of $\tau_{\text {int }}^{p}$ as a function of $\lambda$. Depending on the other parameters, the autocorrelation times were observed to increase or even decrease slightly as $\lambda$ is increased. This behavior can be ascribed to the exact treatment of the fermion degrees of freedom. As we are not sampling the hopping process of the single electron considered here, no autocorrelations due to the resulting reaction of the harmonic oscillators to the electronic motion (see Sec. $\mathrm{VB}$ ) can occur. Moreover, even if we would sample the electronic degrees of freedom in the QMC simulation, these autocorrelations would still be strongly reduced as long as we use the Lang-Firsov transformed model. This is a consequence of the fact that the large displacements of the oscillators in the presence of an electron are explicitly contained in the Hamiltonian (6). Finally, as the number of lattice sites is varied, $\tau_{\text {int }}^{p}$ remains constant in units of sweeps for our single-electron simulations. We also determined the autocorrelation times for observables such as, e.g., the kinetic energy. Although $\tau_{\text {int }}$ is smaller for electronic observables, the problem still exists, and the determination of the autocorrelation times for various parameter sets is vital to obtain reliable results. This usually requires very long QMC runs and a lot of CPU time.

As indicated in Sec. VB the autocorrelations which arise from the structure of the bosonic action $S_{\mathrm{b}}$ [see Eq. (32)] may be overcome by a transformation to the normal modes of the system. Here we represent the bosonic action $S_{\mathrm{b}}$ in terms of its normal modes along the imaginary time axis. This allows us to sample completely uncorrelated phonon configurations. In combination with the reweighting method the fermion degrees of freedom are treated exactly, so that our QMC method is indeed free of any autocorrelations. This greatly simplifies calculations, since it makes the usual binning analysis (to determine the autocorrelation times) obsolete and, more importantly, leads to significantly shorter simulation times.

All this can be achieved with the simple but effective idea of a transformation to principal components (PCs). To this end let us recall the form of the bosonic action given by Eq. (33) which can also be written as

$$
S_{\mathrm{b}}=\sum_{i} \boldsymbol{p}_{i}^{\mathrm{T}} A \boldsymbol{p}_{i}=\sum_{i} \boldsymbol{p}_{i}^{\mathrm{T}} A^{1 / 2} A^{1 / 2} \boldsymbol{p}_{i}=: \sum_{i} \boldsymbol{\xi}_{i}^{\mathrm{T}} \cdot \boldsymbol{\xi}_{i}
$$

with the PCs $\boldsymbol{\xi}_{i}=A^{1 / 2} \boldsymbol{p}_{i}$, in terms of which the bosonic weight takes the simple Gaussian form

$$
w_{\mathrm{b}}=e^{-\Delta \tau \sum_{i} \boldsymbol{\xi}_{i}^{\mathrm{T}} \cdot \boldsymbol{\xi}_{i}} .
$$

The QMC can now be performed directly in terms of the new variables $\xi$. To calculate observables we have to transform back to the phonon momenta $p$ using the matrix $A^{-1 / 2}$. Comparison with Eq. (33) shows that instead of the ill-conditioned matrix $A$ we now have the ideal structure that we can easily generate exact samples of a Gaussian distribution. In terms of the new coordinates $\xi$, the probability distribution can be sampled exactly, e.g., by the Box-Müller method. ${ }^{69}$ In contrast to a standard Markov chain MC simulation, every new configuration is accepted, and measurements of observables can be made at each step.

From the definition of the PCs it is obvious that an update of a single variable $\xi_{i, \tau}$, say, actually corresponds to a change of all phonon coordinates $p_{i, \tau^{\prime}}, \tau^{\prime}=1, \ldots, L$. Thus, in terms of the original phonon coordinates $p_{i}$, the updating loses its local character. As a consequence, the sequential updating of the Trotter time slices, which we mentioned in Sec. $\nabla$ can no longer be exploited to reduce the numerical effort for the evaluation of the fermionic weight. However, in combination with the reweighting method, the latter is only calculated when measurements of observables are made. The enormous advantage of the PCs, leading to completely uncorrelated phonon configurations, clearly outweighs this drawback. Nevertheless, this restriction has to be kept in mind when considering possible extensions to many-electron systems. Apart from this, the $\mathrm{PC}$ representation can also be applied to the more general case of more than one electron, since the bosonic action [Eq. (46]), on which the transformation relies, remains unchanged]. This even holds for the case of more general models including, e.g., spin-spin or Hubbard-type interactions, as long as the phonon operators enter in the same form as in the Holstein model.

Another important point is the combination of the PCs with the reweighting method. Using the latter, the changes to the original momenta $p$, which are made in the simulation, do not depend in any way on the electronic degrees of freedom. Thus we are actually sampling a set of $N$ independent harmonic oscillators, as described by the purely bosonic action $S_{\mathrm{b}}$. The crucial requirement for the success of this method is the use of the LangFirsov transformed model, in which the polaron effects are separated from the zero-point motion of the oscillators around their current equilibrium positions.

Finally, for the untransformed model, Eq. (1), the bosonic action can be obtained from Eq. (33) by replacing $p$ with $x$ (see Sec. $\nabla \mathrm{A}$ ) and a transformation to PCs could also be used. However, as discussed in Sec. VI without the Lang-Firsov transformation, the reweighting procedure fails. Consequently, using the standard approach, the phonon coordinates $x$ would depend on the electronic degrees of freedom, and this makes exact sampling impossible for the untransformed model.

\section{RESULTS: VPA}

In order to test the validity of VPA we calculated the total energy [Eq. (18)] and the quasiparticle weight 

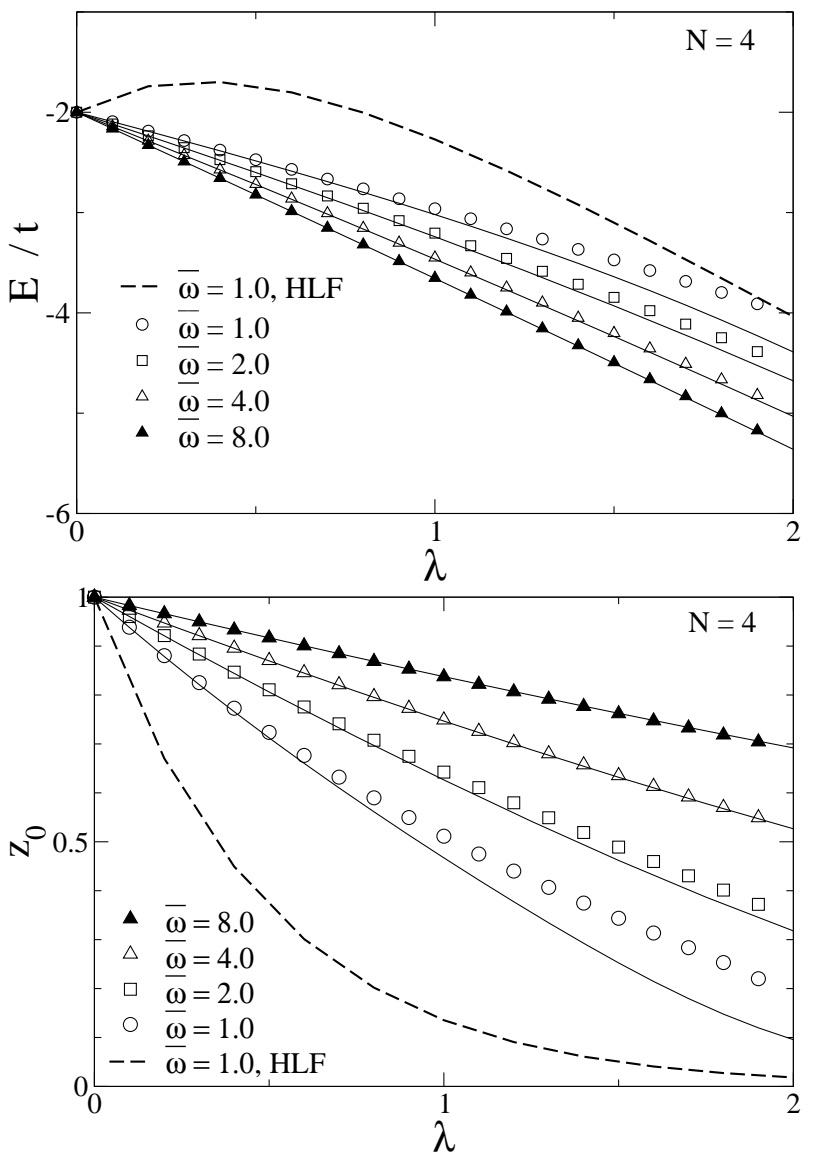

FIG. 2: Total energy $E$ (top) and quasiparticle weight $z_{0}$ (bottom) as functions of the electron-phonon coupling $\lambda$ for different values of the phonon frequency $\bar{\omega}$. Symbols correspond to VPA results, while full lines represent exact $T=0$ data obtained with the Lanczos method (Ref. 70). Dashed lines are results of the HLF approximation.

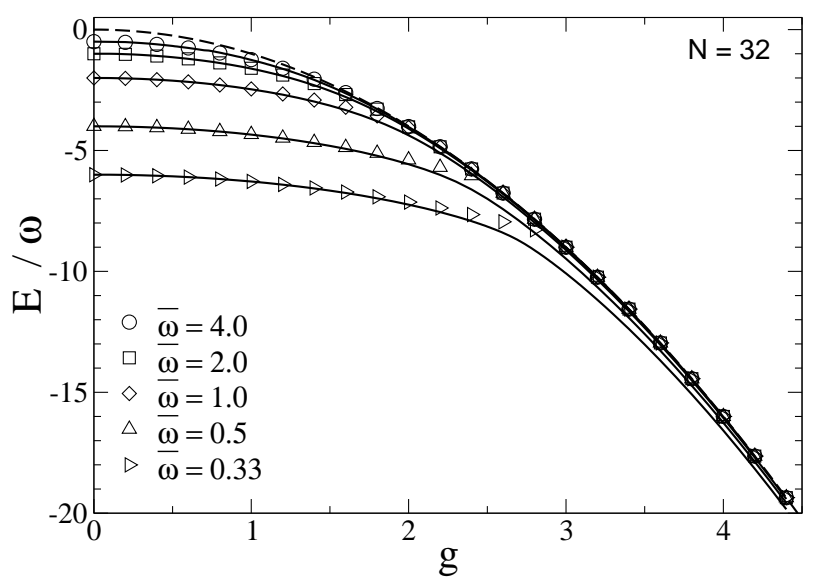

FIG. 3: Total energy $E$ as a function of the electron-phonon coupling $g$ (see text) for different values of the phonon frequency $\bar{\omega}$. Symbols correspond to VPA results, while full lines represent data obtained with the globallLocal method (Ref. 57). The dashed line represents the atomic-limit result $(\bar{\omega}=\infty)$.
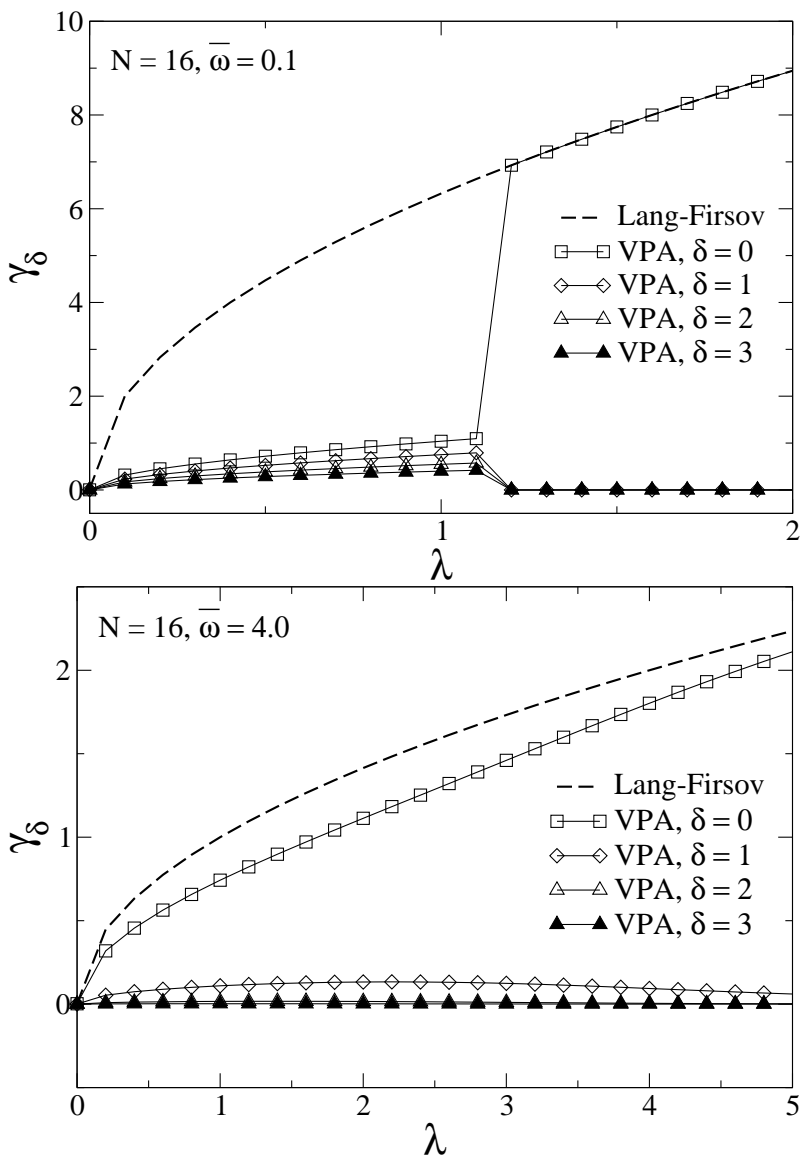

FIG. 4: Polaron-size parameter $\gamma_{\delta}$ as a function of the electron-phonon coupling $\lambda$ for various distances $\delta$. The parameter $\gamma$ of the standard Lang-Firsov transformation (see Sec. IIII) is also shown.

[Eq. [23)] on a cluster of four sites for various phonon frequencies $\omega$ and compared the results with those of Marsiglio obtained by Lanczos diagonalization $\frac{70}{\underline{\omega}}$ The comparison is depicted in Fig. 2 The values of $\bar{\omega}$ have been chosen to lie in the nonadiabatic regime $\bar{\omega} \geq 1$ where the zero-phonon approximation of the VPA is sensible. The overall agreement is strikingly good. Minor deviations from the exact results increase with decreasing phonon frequency. For the smallest frequency shown, $\bar{\omega}=1.0$, the curve for the HLF approximation is also depicted. It reveals that VPA represents a significant improvement over the HLF approximation, underlining the importance of the extended polaron cloud.

The comparison with exact results obtained with Lanczos was restricted to small clusters with $N=4$ in order to achieve convergence with respect to the number of phonon states included in the calculation (see Sec. III). To further scrutinize the accuracy of the VPA we also compare the results of the latter for the total energy with the variational global-local method which has been shown to give reliable results over a large range of parameters 57 We chose $N=32$ for which finite-size effects are already very small (see Sec. IX]). Moreover, following Romero et 
al.,$\frac{57}{1}$ in Fig. 3 we plot $E / \omega$ over $g$ with $g=\sqrt{\lambda W /(2 \omega)}$. Similar to the case $N=4$ shown in Fig. 2 we find a very good agreement for large values of $\bar{\omega}$ over the whole range of electron-phonon coupling, whereas for smaller $\bar{\omega}$ the VPA results begin to bend away from the correct curve and collapse to the strong-coupling, atomic-limit result for large $g$. We would like to point out that the maximum electron-phonon coupling strength in Fig. 3 corresponds to $\lambda \approx 40$ (for $\bar{\omega}=4.0$ ), in contrast to Fig. 2 where $\lambda \leq 2$. Figures 2 and 3 reveal that in the nonadiabatic regime $\bar{\omega} \gg 1$ VPA yields a very good agreement with the exact data and the Global-Local method even in the intermediate and strong-coupling regime. This behavior can easily be understood considering the assumptions of the VPA. The zero-phonon approximation becomes exact in the nonadiabatic limit $\bar{\omega} \rightarrow \infty$, where the energies of phonon excitations are too high to have an effect on the ground state. Finally, we would like to mention the possibility of comparing the VPA with the QMC results presented in the following section. This has been done for a variety of parameters, but we have found that it is difficult to distinguish between deviations due to the shortcomings of the VPA and due to temperature effects in the QMC results. Consequently, we have decided to confront the VPA with another approved ground-state method, namely, the global-local method, which gives a much clearer picture.

In Fig. 4 we show results for the variational displacement fields $\gamma_{\delta}$, which give us a measure for the size of the polaron. For $\bar{\omega}=0.1$ we see an abrupt crossover from a large to a small polaron at $\lambda \approx 1.2$. For smaller values of the coupling, the electron induces lattice distortions at neighboring sites even at a distance of more than three lattice constants. Above $\lambda \approx 1.2$ we have a mobile small polaron extending over a single site only. In contrast, for a larger value of the phonon frequency $\bar{\omega}=4.0$, there is no crossover and we have a somewhat extended (large) polaron even for large values of $\lambda$. The same behavior has been found by Marsiglio 36 who determined the correlation function $\left\langle n_{i} x_{i+\delta}\right\rangle$ by Lanczos diagonalization for a restricted phonon basis. Within VPA we have the relation $\left\langle n_{i} x_{i+\delta}\right\rangle=\gamma_{\delta}$. The main difference is that in Marsiglio's results, the crossover to a small polaron for $\bar{\omega}=0.1$ occurs at a smaller value of the coupling $\lambda \approx 1$. Nevertheless, the simple VPA reproduces the main characteristics of the transition of the Holstein polaron as the coupling strength $\lambda$ is increased. Finally Fig. 4 also shows the result for the parameter $\gamma$ of the standard Lang-Firsov transformation (see Sec. III). For $\bar{\omega}=0.1$, the curves for $\gamma$ and $\gamma_{\delta=0}$ are identical above the critical value $\lambda \approx 1.2$. This is not surprising since, in this regime, we have a small polaron extending over a single site only, which is well described by the local Lang-Firsov transformation defined in Eq. (5). For larger values of the phonon frequency (see Fig. (4), $\gamma$ and $\gamma_{0}$ do not coincide above a critical value of the coupling, but the difference vanishes as $\lambda \rightarrow \infty$. In contrast to the adiabatic regime, the polaron remains an extended object up to very large values of the coupling, so that the local ansatz of the Lang-Firsov transformation does then not provide the correct description for finite values of $\lambda$ (see also Ref. 62).

\section{RESULTS: QUANTUM MONTE CARLO}

As our approach is based on a discretized imaginary time, it is important to study the convergence of any results with increasing number of time slices, $L$, which determines the error due to the Suzuki-Trotter approximation of Eq. (25). $L$ was chosen such that systematic errors are smaller than the statistical errors of the results. For all observables considered here we have found the usual $(\Delta \tau)^{2}$ dependence of the Suzuki-Trotter error. Depending on the phonon frequency $\bar{\omega}$ we have found values of $\Delta \tau=1 / 30$ (for $\bar{\omega} \lesssim 1$ ) and $\Delta \tau=1 / 40$ (for $\bar{\omega}>1$ ) to be sufficient even for the most accurate results of this paper. Moreover, as indicated in Fig. 9] error bars for the QMC data presented are always smaller than the symbols used in the figures and are therefore not shown. Finally, lines connecting data points obtained with QMC in Figs. 911] are guides to the eye only.

To test our QMC algorithm we have performed several comparisons with other methods. First, we have checked that the QMC reproduces the exact results obtained with Lanczos on a four-site cluster. Apart from temperature effects, an excellent agreement has been found for several different values of the phonon frequency. Second, as the QMC results are all for finite temperature, we have also compared them with an exact solution for the two-site system, which is valid for arbitrary temperature. We have found a perfect agreement over the whole range of values for $\beta, \bar{\omega}$, and $\lambda$, and can therefore exclude the possibility of any systematic errors.

\section{A. Kinetic energy}

We begin our discussion of the results with the kinetic energy of the electron, given by Eq. (38), which has previously been calculated by several authors 22.23 .25 .43 .49 .57 .65 In Fig. 5 we show results for $E_{\mathrm{k}}$ on a 32 -site cluster, with $\beta t=10$ and for several values of the phonon frequency. While for small values of $\bar{\omega}$ there is a rapid decrease of the absolute value of the kinetic energy in the vicinity of $\lambda=1$, the cross over becomes smoother as $\bar{\omega}$ increases. This agrees with the findings of previous studies and resembles closely the behavior of the total energy discussed above. For large values of $\lambda$ and $\bar{\omega} \lesssim 1$ we find $E_{\mathrm{k}} \sim \lambda^{-1}$ as predicted by small-polaron theory ${ }^{71}$ This contrasts strongly with the behavior of the quasiparticle weight $z_{0}$ [see Fig. 2 (b)] which decreases much faster and is exponentially suppressed in the small-polaron regime ${ }^{48} \mathrm{As}$ pointed out by Fehske et al. 59 for the case of the Holstein model, the quasiparticle weight is exactly the inverse of the ratio $m_{\mathrm{eff}} / m$ where $m_{\mathrm{eff}}$ and $m$ denote the effective 


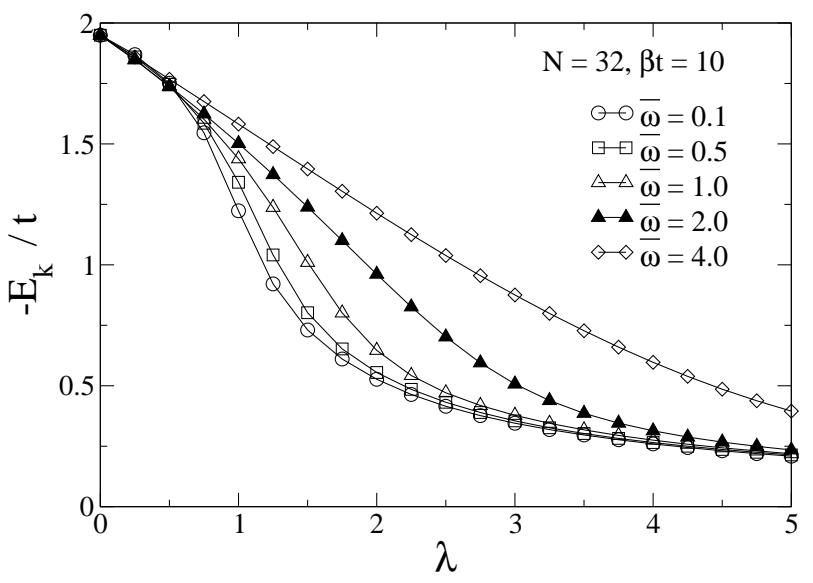

FIG. 5: Negative kinetic energy $E_{\mathrm{k}}$ as a function of the electron-phonon coupling $\lambda$ for various values of the phonon frequency $\bar{\omega}$.

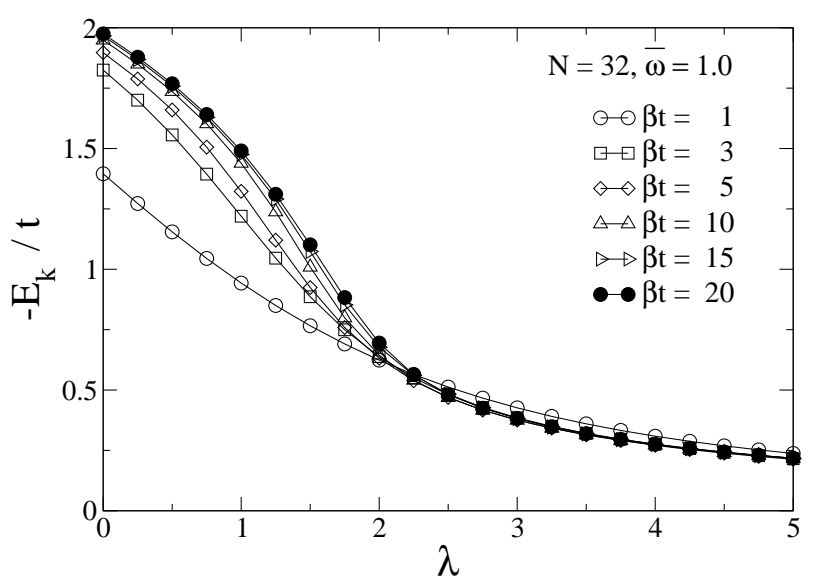

FIG. 6: Negative kinetic energy as a function of the electronphonon coupling $\lambda$ for various values of the inverse temperature $\beta$ for various values of the phonon frequency $\bar{\omega}$.

and free mass of the electron, respectively. Hence, in the small-polaron regime, the effective mass increases exponentially, while the kinetic energy still has a finite value. We ascribe this behavior to the undirected motion of the electron inside the phonon cloud, which gives rise to a nonzero kinetic energy even for large values of $\lambda$. However, since the polaron bandwidth is exponentially narrowed with increasing $\lambda$, the polaron is almost localized.

To study the influence of temperature we have calculated the kinetic energy for a system of 32 sites, with $\bar{\omega}=1.0$ and for various values of $\beta t$ (see Fig. [6). As $\beta t$ increases, $\left|E_{\mathrm{k}}\right|$ increases for $\lambda \lesssim 2$. However temperature effects are obviously very small in the strong-coupling regime. For $\beta t=1,\left|E_{\mathrm{k}}\right|$ decays in a smooth way as $\lambda$ is increased, while for lower temperatures we find the typical rather abrupt crossover near $\lambda=1$, as in Fig. 5 De Raedt and Lagendijk ${ }^{23}$ have calculated the kinetic energy for the same set of parameters using their QMC method. However, the lowest temperature they could reach was

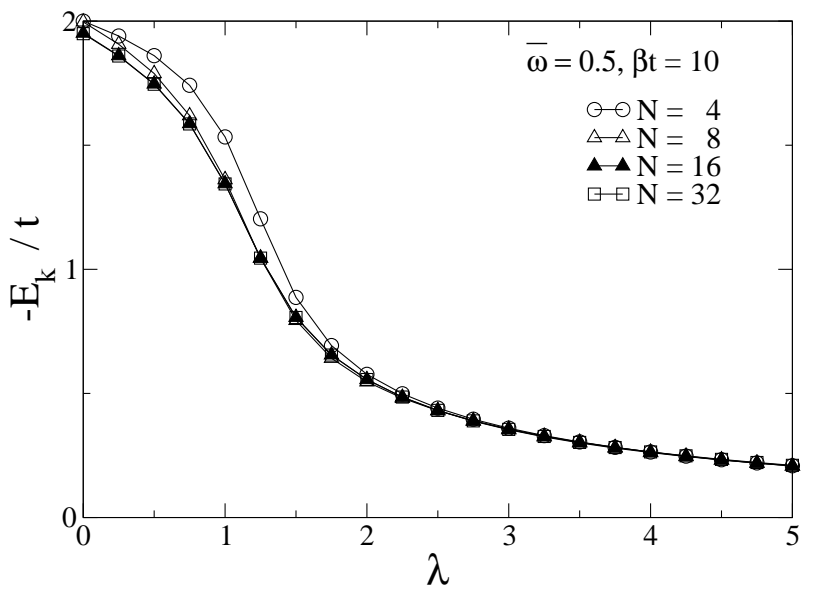

FIG. 7: Negative kinetic energy as a function of the electronphonon coupling $\lambda$ for different numbers of lattice sites $N$.
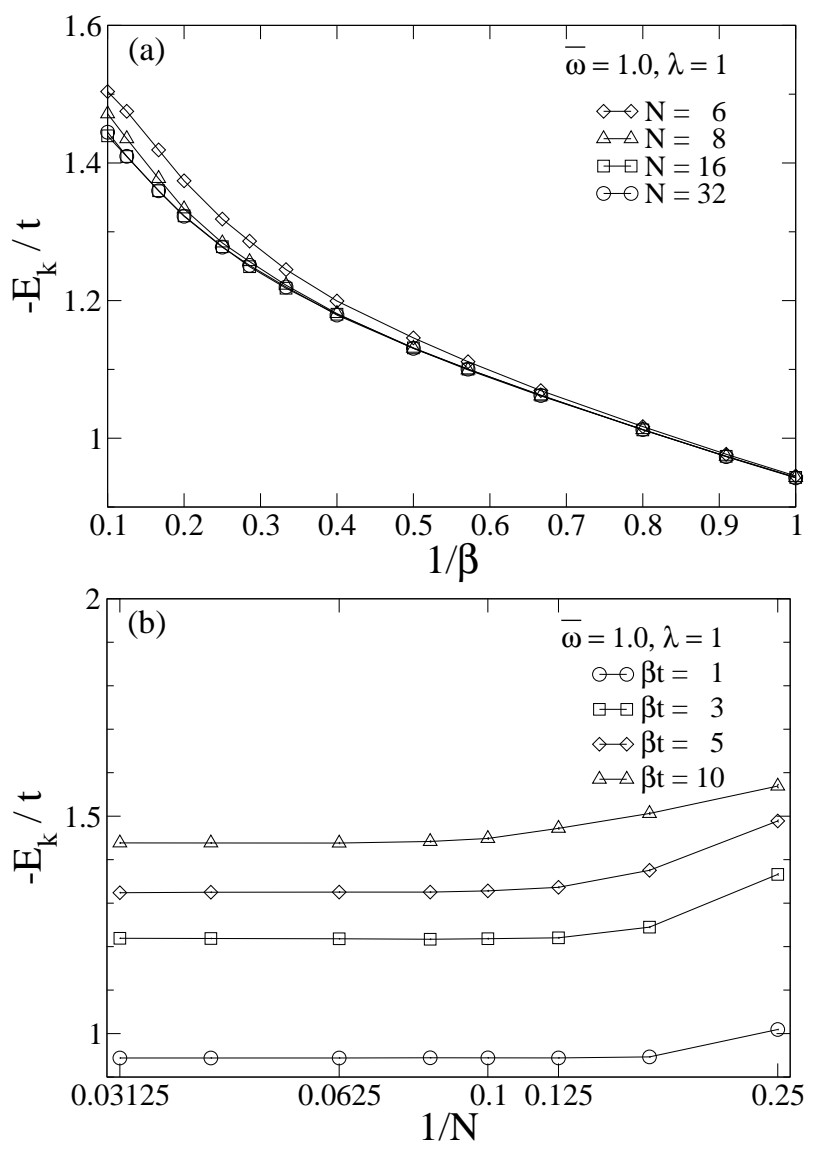

FIG. 8: Negative kinetic energy (a) as a function of the inverse temperature $\beta$ and (b) as a function of the inverse of the number of lattice sites $N$.

$\beta t=5$ which, according to Fig. [6 is still quite different from the ground-state result. Moreover, their calculations did not include dynamical effects of the phonon degrees of freedom. As a consequence, for $\beta t=1$, they do not obtain the correct behavior of the kinetic energy as a function of $\lambda$. Finally, Romero et al $\stackrel{57}{5}$ and Jeckelmann 
and White $\mathrm{e}^{49}$ calculated the kinetic energy for $T=0$ on a 32-site cluster and for an infinite system, respectively. Their results are in a good agreement with our findings, although small deviations due to temperature and finitesize effects are visible. Nevertheless, we can conclude from Fig. [6] that a value of $\beta t=10$ should be sufficient to obtain results which are representative of the ground state.

We now turn our attention to finite-size effects. In Fig. 7 we show the kinetic energy for $\bar{\omega}=0.5, \beta t=10$, and for various number of lattice sites. For $N \geq 16$ the results for $E_{\mathrm{k}}$ are well converged over the whole range of $\lambda$ and finite-size effects are very small. This agrees with the findings of other authors ${ }^{23.36 .42}$ Figure 8 shows the kinetic energy as a function of temperature, for $\bar{\omega}=1.0$ and various numbers of lattice sites $N$. Moreover we chose $\lambda=1$, as the influence of the system size is largest in the cross over regime. Figure 8(a) clearly demonstrates that finite-size effects are most pronounced at low temperatures, while they are completely smeared out at higher temperatures, since high-temperature properties are determined by integral quantities, such as energy moments $\left\langle E^{\nu}\right\rangle$, which have a small size dependence, while lowtemperature features are governed by energetically lowlying eigenvectors. To further illustrate the influence of the system size, we plot in Fig. 8 (b) the negative kinetic energy as a function of $1 / N$ again for $\bar{\omega}=\lambda=1$ and for various values of $\beta$. As before, error bars are smaller than the symbol size, but due to the very high accuracy of the data, the systematic errors due to the finite number of Trotter slices $L$ are comparable to the statistical errors. The results show that very good convergence with respect to the number of lattice sites is achieved for rather small $N$. In fact, for the highest temperature shown $(\beta t=1)$, the line connecting the data points becomes vertical already at $N=8$, while for $\beta t=10$ convergence is reached for $N=16$. Hence, if we consider these findings in the context of the usual finite-size scaling analysis where one plots the data as a function of a suitably chosen power of $1 / N$ and extrapolates to the infinite system (i.e., $1 / N \rightarrow 0$ ), we have here the special case of a linear dependence with zero slope at large enough $N$. Thus, in contrast to the half-filled Holstein model of spinless fermion, for which a finite-size analysis has been performed by two groups, ${ }^{34.72}$ we merely find that the results converge within the accuracy of our calculations already for rather small systems.

\section{B. Total energy}

Next we consider the total energy $E$, given by Eq. (41). In Fig. 9 we present the total energy for a cluster of 32 sites and various values of the phonon frequency. Finite temperature effects increase as we approach the low-frequency regime, and for $\bar{\omega}=0.1$ we clearly see a strong deviation from the ground-state result $E=-2 t$ for $\lambda=0$. The frequency-dependence of the temperature

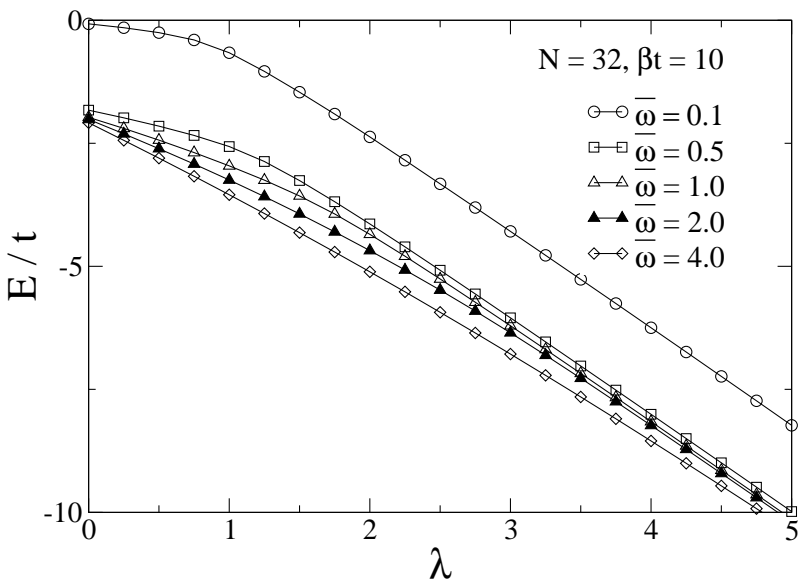

FIG. 9: QMC results for the total energy $E$ as a function of the electron-phonon coupling $\lambda$ for various values of the phonon frequency $\bar{\omega}$. Here and in subsequent figures lines are guides to the eye, and errorbars for the QMC data are smaller than the symbols shown.

effects can easily be understood if we consider the exact result for the kinetic energy of $N$ independent harmonic oscillators

$$
E_{\mathrm{k}, \mathrm{ph}}=\frac{\omega}{2} \sum_{i}\left\langle p_{i}^{2}\right\rangle=\frac{N \omega}{2}\left(\frac{1}{2}+\frac{1}{e^{\beta \omega}-1}\right),
$$

which is identical to the second term in Eq. (41). For low temperatures we have $\left\langle p^{2}\right\rangle \approx 0.5+e^{-\beta \omega}$, with a correction to the ground-state value of 0.5 that increases with decreasing $\omega$. These temperature effects on $E$ due to the oscillator energy do not depend on $\lambda$ [see Eq. [47]] and therefore shift the total energy curve by the same amount for all values of the coupling. A comparison with the discussion of the kinetic energy reveals that temperature effects are much smaller for other observables due to the absence of the strongly temperature-dependent phonon energy terms $P_{\mathrm{p}}$ and $E_{\mathrm{ph}}^{\prime}$ [see Eqs. (11) and (41)].

The dependence on $\bar{\omega}$ agrees well with existing

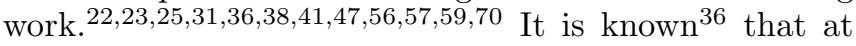
zero temperature and for small values of the phonon frequency, $\bar{\omega} \lesssim 0.5$, the total energy displays a rather sharp transition around $\lambda \approx 1$, where the cross over from a large to a small polaron occurs. In ED studies of small clusters ${ }^{36}$ a kink in $E$ has been observed, which is smeared out in the finite-temperature QMC results. Nevertheless, we observe the same rounding of the energy curve with increasing $\bar{\omega}$ (Ref. 36). As discussed by Marsiglio 36 the kink in the total energy is merely a finitesize effect. As the system size increases the discontinuity disappears, in accordance with the fact that the ground state of the Holstein polaron is an analytic function of the coupling parameter $\lambda$ (Ref. 64).

Finally, it is interesting to note that in contrast to the kinetic energy $-E_{\mathrm{k}}$, which shows a sharp decrease near $\lambda=1$ in the adiabatic regime (see, e.g., Fig. 15), the total energy does not change significantly. As discussed for 
the two-dimensional case by Kornilovitch 25 this can be explained as follows. For small ratios $\omega / t$, the phonon energy associated with the term $P$ of Hamiltonian (1) is small and the system is governed by the balance of the electronic kinetic energy and the energy due to the electron-phonon coupling. In the transformed model, the latter is given by $E_{\mathrm{p}}$ as defined by Eq. (8). When the ratio of the two energies approaches unity (equivalent to $\lambda=1$ ), it becomes energetically favorable for the electron to localize (losing kinetic energy) and increase its potential energy. This leads to finite displacements of the oscillators in the vicinity of the electron and increases the potential energy of the phonons. Hence, near $\lambda=1$ the energy of the system is redistributed from kinetic to potential energy so that $E$ remains almost unchanged. This is exactly what we see in Fig. [5]

\section{Momentum distribution and oscillator momenta}

Following Zhang et al ${ }^{50}$ we also calculated the momentum distribution $n(k)$, given by Eq. (42), for different wave vectors $k$ (Fig. 10). To compare with their $\mathrm{DMRG}^{73}$ results we chose the same parameters $N=6$ and $\bar{\omega}=1.0$. Moreover, we took $\beta t=10$ since the calculations of Zhang et al. were for the ground state. For $\lambda=0$ the ground state has momentum $k=0$, so we have $n(0)=1$ and $n(k \neq 0)=0$. With increasing coupling $n(0)$ decreases in a way similar to the kinetic energy (cf. Fig. [5), while $n(k)$ for $k \neq 0$ increases. In the strong-coupling limit $\lambda \rightarrow \infty, n(k)$ approaches the value $1 / N=1 / 6$ for all $k$. This is a simple consequence of the localization of the electron for $\lambda=\infty$. Although the curve for $k=0$ looks very similar to the results of Zhang et al. we find a slightly stronger decrease of $n(0)$ in the intermediate coupling regime. This deviation is no temperature effect of our QMC method but probably originates from the fact that Zhang et al. obtained their results for $n(0)$ by integrating over an approximate spectral function.

In Sec. VI we mentioned that, within the Lang-Firsov approach, the phonon degrees of freedom only show a weak dependence on the electron-phonon coupling, in contrast to the standard approach, where the average oscillator coordinate $\langle x\rangle$ increases strongly with $\lambda$ due to the displacement in the presence of an electron. The weak dependence of the vibrational energy of the local oscillator, which is proportional to $\left\langle p^{2}\right\rangle$, on $\lambda$ is shown in Fig. 11] For $\lambda=0$ we have the result $\left\langle p^{2}\right\rangle=$ $0.5+[\exp (\beta \omega)-1]^{-1}[$ see Eq. [47])] for a free oscillator. In the intermediate coupling regime, $\left\langle p^{2}\right\rangle$ takes on a minimum, corresponding to a reduction of merely $4 \%$ and approaches the value for $\lambda=0$ again in the strong-coupling limit. As the Lang-Firsov transformation does not affect the phonon momenta $p$ (see Sec. III), the result for $\left\langle p^{2}\right\rangle$ as a function of $\lambda$ is the same in the untransformed Holstein model. However, the significant advantage of the proposed method is that the phonon momenta are

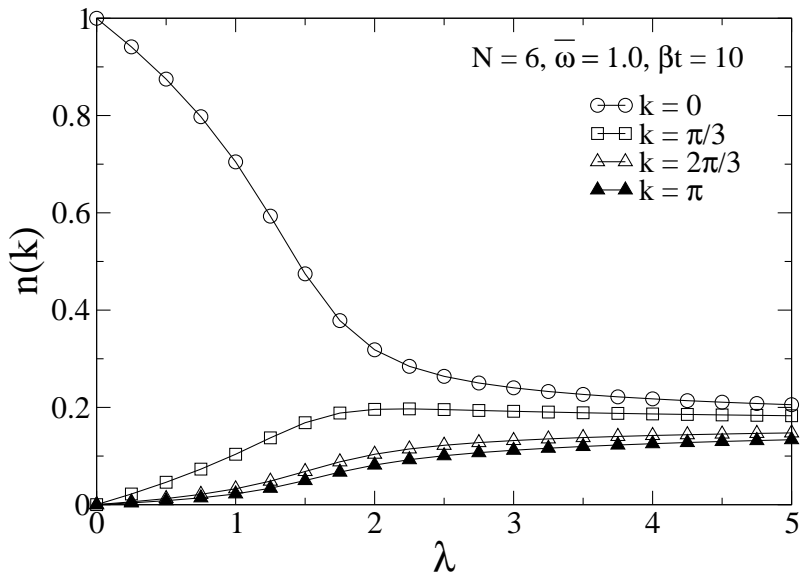

FIG. 10: Momentum distribution $n(k)$ as a function of $\lambda$ for various wave vectors $k$.

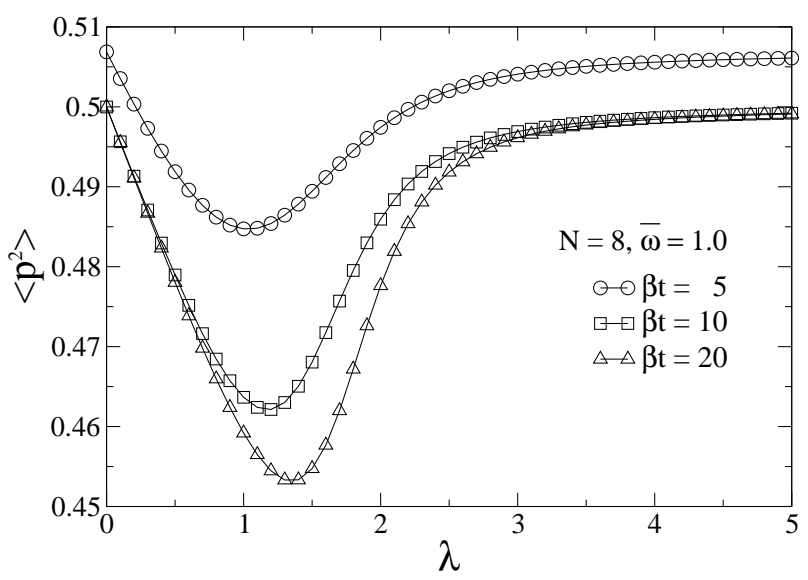

FIG. 11: Mean square of the phonon momentum $p$ as a function of the electron-phonon coupling $\lambda$.

sampled instead of the coordinates $x$. Thus the probability distribution associated with the degrees of freedom to be sampled has only a small variance compared to the standard method, which makes the simulations much more effective. The dependence of $\left\langle p^{2}\right\rangle$ on the coupling strength $\lambda$ and the temperature has first been studied by Ranninger and Thibblin ${ }^{41}$ for the two-site polaron problem. For such a small system, the minimum of $\left\langle p^{2}\right\rangle$ is even more pronounced, while for larger systems the average effect of the electron on a local oscillator is more and more washed out. Ranninger and Thibblin ${ }^{41}$ ascribed the deviation of the vibrational energy from the free-oscillator result to anharmonic effects, which are visible only at low enough temperatures. This can clearly be seen in Fig.11] where the minimum of $\left\langle p^{2}\right\rangle$ becomes less pronounced and is shifted to smaller values of $\lambda$ as the temperature increases. 


\section{Performance}

We conclude this section with a discussion of the performance of the QMC approach. From the results presented above it is obvious that the method enables us to study a very wide range of parameters. Hence, for example, we have performed calculations for $0.1 \leq \bar{\omega} \leq 4.0$ (see Fig. (5). Simulations in the adiabatic regime would be extremely difficult within the standard approach, since the autocorrelation times grow as $(\omega \Delta \tau)^{-2}$. However, in materials such as the manganites, the frequencies of the relevant phonon modes are known to be small $(\bar{\omega} \lesssim 0.5$, see, e.g., Ref. 22) so that our method could represent an important step forward towards the simulation of electronphonon models with realistic parameters. Also, we are able to reach very low temperatures $\beta t \leq 20$ and clusters large enough to avoid finite-size effects with modest computational effort. Another key advantage is that the method becomes more and more efficient as the coupling strength $\lambda$ increases, which is due to the use of the LangFirsov transformation. In our results we find that statistical errors of expectation values of phonon operators are larger than, e.g., the errors of the kinetic energy. Finally, the errors increase slightly as we approach the adiabatic and/or low-temperature regime $\bar{\omega} \rightarrow 0$ and $\beta t \rightarrow \infty$, respectively.

To demonstrate the efficiency of our method we give some figures for the CPU time of the simulations. A typical QMC run for 32 lattice sites, $\beta t=5, \bar{\omega}=1.0$, and $\lambda \approx 1$ (i.e., near the small-polaron crossover) only takes $5 \mathrm{~min}$ of $\mathrm{CPU}$ time on a $650 \mathrm{MHz}$ Pentium III PC. For such a run relative errors of, for example, the kinetic energy are less than $1.0 \%$. Away from the crossover point, the same accuracy can be obtained within a few seconds. For $\beta t=10$, the temperature used in most of the calculations presented in this paper, a QMC run with $\lambda$ near the crossover value and with similar statistical errors as mentioned above takes about $80 \mathrm{~min}$ of CPU time. Hence, although not as efficient as the specialized one-electron methods, ${ }^{22.23 .24 .25 .31 .32}$ our approach significantly reduces the numerical effort compared to previous methods which were often run on supercomputers and did not reach the parameters (low temperature and small phonon frequency) and accuracy of our simulations.

\section{CONCLUSIONS}

We have presented a simple variational approach to the Holstein model, which incorporates an extended LangFirsov transformation. This approach is easily applicable to infinite systems and represents a marked improvement over the standard small-polaron approximation, which is only useful in the nonadiabatic, strong-coupling regime.

More importantly, we have introduced an exact QMC method for the Holstein model, which is based on the standard Lang-Firsov transformation of the Hamiltonian. The phonon momenta are represented in terms of princi- pal components, which enables us to sample completely uncorrelated configurations, while the electronic degrees of freedom are taken into account exactly by use of a reweighting method for calculating observables. Thereby, we avoid the numerically expensive evaluation of the electronic weights in the updating process. The present approach can be applied for a wide range of parameters with relatively small computational effort. In particular, efficient simulations can be performed in the adiabatic regime $\bar{\omega}<1$, which is of special interest in connection with materials such as the manganites. In the onedimensional case considered here, a sign problem resulting from the Lang-Firsov transformation on small systems has been found to have only a small effect on the statistics. Tests have been presented in the one-electron case and reveal that the method reproduces Lanczos diagonalization results in the regime where the latter are applicable, namely, for very small systems, small to moderate electron-phonon coupling and for sufficiently large phonon frequency. Moreover, a satisfactory agreement with other methods has been found. Owing to the exact treatment of the electronic degrees of freedom and the sampling of the phonons, the method is free of any autocorrelations. The use of the Lang-Firsov transformation, which is essential for the applicability of the reweighting method, substantially improves the statistics, allowing for very accurate results.

Despite the great computational efficiency of our method compared to the standard approach, even faster methods exist. For example, the QMC simulations of de Raedt and Lagendijk ${ }^{22,23,24}$ and Kornilovitch ${ }^{25,31.32}$ seem to be numerically faster due to the analytic integration over the phonon degrees of freedom which significantly reduces statistical errors. However, both methods are restricted in their applicability as discussed in Sec. [ In particular, an extension to many-electron systems seems impossible, since simulations will be restricted by a severe minus sign problem similar to other world-line methods. In contrast, the method presented here is not restricted to the single-electron limit in principle, although some modifications will be necessary. As pointed out in previous sections, most of the ideas proposed here, such as the use of the transformed model, the reweighting method, and the PC representation, remain unchanged if we consider more than one electron. The required modifications concern mainly the fermionic weight $w_{\mathrm{f}}[$ Eq. [29] ]. There is a Hubbard-like interaction term coming from the LangFirsov transformation (see Sec. III), and the one-electron basis states used here (Eq. (31) have to be replaced by the corresponding set of many-electron states. Since the number of such basis states, and thereby the linear dimension of the matrices $\Omega, \kappa$, and $D$ (see Sec. $\overline{\nabla A}$ ), grows exponentially with the system size, an exact treatment of the fermion degrees of freedom will become increasingly difficult. Consequently, a more refined approach based on, e.g., the use of determinant methods will be required. For the bipolaron problem of two electrons with opposite spin, on which work is currently in progress, 
the computational effort can be significantly reduced by exploiting the conservation of the total quasimomentum (see, e.g., Ref. 28), leading to a computer time that grows with the cube of the system size. The more general case of, e.g., a quarter-filled band corresponding to the colossal magnetoresistance regime of the manganites, requires further consideration, and the effect of the sign problem remains to be investigated. Moreover, the performance of such an approach has to be compared to existing manyelectron QMC methods for the Holstein model. Finally, the method can be generalized to more complicated models including, e.g., a coupling of the electrons to local spins as in the Kondo or double-exchange model for the manganites.

\section{Acknowledgments}

This work was partially supported by the Austrian Science Fund (FWF), project No. P15834. M.H. is grateful to the Austrian Academy of Sciences for financial support. We would like to acknowledge helpful discussions with Markus Aichhorn, Holger Fehske, Winfried Koller, and Alexander Prüll. We would also like to thank Frank Marsiglio and Aldo Romero for providing us with some of the data presented in this paper.
* Electronic address: hohenadler@itp.tu-graz.ac.at

1 Y. Bar-Yam, J. Mustre de Leon, and A. R. Bishop, eds., Lattice Effects in High Temperature Superconductors (World Scientific, Singapore, 1992).

2 D. M. Edwards, Adv. Phys. 51, 1259 (2002).

3 A. J. Millis, R. Mueller, and B. I. Shraiman, Phys. Rev. B 54, 5405 (1996).

${ }^{4}$ E. Dagotto, S. Yunoki, and A. Moreo, in Physics of Manganites, edited by T. A. Kaplan and S. D. Mahanti (Kluwer Publishing, 1999), p. 39.

5 T. Holstein, Ann. Phys. (N.Y.) 8, 325; 8, 343 (1959).

${ }^{6}$ H. Froehlich, H. Pelzer, and S. Zienau, Philos. Mag. 41, 221 (1950).

7 W. P. Su, J. R. Schrieffer, and A. J. Heeger, Phys. Rev. Lett. 42, 1698 (1979).

8 R. Blankenbecler, D. J. Scalapino, and R. L. Sugar, Physical Review D 24, 2278 (1981).

9 D. J. Scalapino and R. L. Sugar, Phys. Rev. B 24, 4295 (1981).

10 G. Levine and W. P. Su, Phys. Rev. B 42, 4143 (1990).

11 G. Levine and W. P. Su, Phys. Rev. B 43, 10413 (1991).

12 P. Niyaz, J. E. Gubernatis, R. T. Scalettar, and C. Y. Fong, Phys. Rev. B 48, 16011 (1993).

13 J. E. Hirsch, D. J. Scalapino, R. L. Sugar, and R. Blankenbecler, Phys. Rev. Lett. 47, 1628 (1981).

14 J. E. Hirsch, R. L. Sugar, D. J. Scalapino, and R. Blankenbecler, Phys. Rev. B 26, 5033 (1982).

15 J. E. Hirsch and E. Fradkin, Phys. Rev. Lett. 49, 402 (1982).

16 E. Fradkin and J. E. Hirsch, Phys. Rev. B 27, 1680 (1983).

17 J. E. Hirsch and E. Fradkin, Phys. Rev. B 27, 4302 (1983).

18 W. von der Linden, Phys. Rep. 220, 53 (1992).

19 R. T. Scalettar, N. E. Bickers, and D. J. Scalapino, Phys. Rev. B 40, 197 (1989).

${ }^{20}$ R. M. Noack, D. J. Scalapino, and R. T. Scalettar, Phys. Rev. Lett. 66, 778 (1991).

21 F. Marsiglio, Phys. Rev. B 42, 2416 (1990).

22 H. De Raedt and A. Lagendijk, Phys. Rev. Lett. 49, 1522 (1982).

23 H. De Raedt and A. Lagendijk, Phys. Rev. B 27, 6097 (1983).

24 H. De Raedt and A. Lagendijk, Phys. Rev. B 30, 1671 (1984).

25 P. Kornilovitch, J. Phys.: Condens. Matter 9, 10675
(1997).

26 R. P. Feynman, Physical Review 97, 660 (1955).

27 H. de Raedt and A. Lagendijk, Z. Phys. B: Condens. Matter 65, 43 (1986).

28 P. E. Kornilovitch and E. R. Pike, Phys. Rev. B 55, R8634 (1997).

29 N. V. Prokof'ev and B. V. Svistunov, Phys. Rev. Lett. 81, 2514 (1998).

30 A. S. Mishchenko, N. V. Prokof'ev, A. Sakamoto, and B. V. Svistunov, Phys. Rev. B 62, 6317 (2000).

31 P. E. Kornilovitch, Phys. Rev. Lett. 81, 5382 (1998).

32 P. E. Kornilovitch, Phys. Rev. B 60, 3237 (1999).

33 E. Berger, P. Valášek, and W. von der Linden, Phys. Rev. B 52, 4806 (1995).

34 R. H. McKenzie, C. J. Hamer, and D. W. Murray, Phys. Rev. B 53, 9676 (1996).

35 P. Sengupta, A. W. Sandvik, and D. K. Campbell, Phys. Rev. B 67, 245103 (2003).

36 F. Marsiglio, Physica C 244, 21 (1995).

37 V. V. Kabanov and D. K. Ray, Phys. Lett. A 186, 438 (1994).

38 A. S. Alexandrov, V. V. Kabanov, and D. K. Ray, Phys. Rev. B 49, 9915 (1994).

39 I. G. Lang and Y. A. Firsov, Sov. Phys. JETP 16, 1301 (1962).

40 G. D. Mahan, Many-particle Physics (Plenum Press, New York, 1990), 2nd ed.

41 J. Ranninger and U. Thibblin, Phys. Rev. B 45, 7730 (1992).

42 F. Marsiglio, Physics Letters A 180, 280 (1993).

43 G. Wellein, H. Roder, and H. Fehske, Phys. Rev. B 53, 9666 (1996).

44 G. Wellein and H. Fehske, Phys. Rev. B 56, 4513 (1997).

45 J. M. Robin, Phys. Rev. B 56, 13634 (1997).

46 M. Capone, W. Stephan, and M. Grilli, Phys. Rev. B 56, 4484 (1997).

47 M. Capone, S. Ciuchi, and C. Grimaldi, Europhys. Lett. 42, 523 (1998).

48 E. Jeckelmann and S. R. White, Phys. Rev. B 57, 6376 (1998).

49 E. Jeckelmann, C. Zhang, and S. R. White, Phys. Rev. B 60, 7950 (1999).

50 C. Zhang, E. Jeckelmann, and S. R. White, Phys. Rev. B 60, 14092 (1999). 
51 A. Weiße, H. Fehske, G. Wellein, and A. R. Bishop, Phys. Rev. B 62, R747 (2000).

52 W. Stephan, Phys. Rev. B 54, 8981 (1996).

53 M. Hohenadler, M. Aichhorn, and W. von der Linden, cond-mat/0306740 (2003), unpublished.

54 J. Bonca, S. A. Trugman, and I. Batistic, Phys. Rev. B 60, 1633 (1999).

55 L. C. Ku, S. A. Trugman, and J. Bonca, Phys. Rev. B 65, 174306 (2002).

56 A. H. Romero, D. W. Brown, and K. Lindenberg, Phys. Rev. B 59, 13728 (1999).

57 A. H. Romero, D. W. Brown, and K. Lindenberg, Phys. Rev. B 60, 4618 (1999).

58 V. Cataudella, G. De Filippis, and G. Iadonisi, Phys. Rev. B 63, 52406 (2001).

59 H. Fehske, J. Loos, and G. Wellein, Phys. Rev. B 61, 8016 (2000).

60 O. S. Barisic, Phys. Rev. B 65, 144301 (2002).

61 O. S. Barisic, cond-mat/0211189 (2002), unpublished.

62 M. Acquarone, M. Cuoco, C. Noce, and A. Romano, Phys. Rev. B 63, 035110 (2001).

63 S. Ciuchi, F. de Pasquale, S. Fratini, and D. Feinberg, Phys. Rev. B 56, 4494 (1997).
64 H. Lowen, Phys. Rev. B 37, 8661 (1988).

65 E. V. L. de Mello and J. Ranninger, Phys. Rev. B 55, 14 872 (1997).

66 G. G. Batrouni and R. T. Scalettar, in Quantum Monte Carlo Methods in Physics and Chemistry, edited by M. P. Nightingale and C. J. Umrigar (Kluwer Academic Publishers, 1998), p. 65.

67 J. N. Kapur and H. K. Kesavan, Entropy Optimization Principles with Applications (Academic Press, 1992).

68 A. C. Davison and D. V. Hinkley, Bootstrap Methods and their Application (Cambridge University Press, 1997).

${ }^{69} \mathrm{~W}$. H. Press, Numerical recipes in Fortran 77, http://www.numrec.com.

70 F. Marsiglio, in Recent Progress in Many-Body Theories, edited by E. Schachinger, H. Mitter, and H. Sormann (Plenum Press, 1995), vol. 4.

71 A. S. Alexandrov and N. F. Mott, Rep. Prog. Phys. 57, 1197 (1994).

72 A. Weiße and H. Fehske, Phys. Rev. B 58, 13526 (1998).

73 The coupling parameter $g$ used in Ref. 50 is related to ours by $\lambda=2 g^{2} /(\omega t)$. 